(C) <2019>. This manuscript version is made available under the CC-BY-NC-ND 4.0 license http://creativecommons.org/licenses/by-nc-nd/4.0/

The definitive publisher version is available online at 10.1016/j.compstruct.2018.11.042 


\title{
Crack identification of functionally graded beams using continuous wavelet transform
}

\author{
Lin-Feng Zhu ${ }^{\mathrm{a}}$, Liao-Liang $\mathrm{Ke}^{\mathrm{a}, \mathrm{b}^{*}}$, Xin-Qun Zhu ${ }^{c}$, Yang Xiang ${ }^{d}$, Yue-Sheng Wang ${ }^{\mathrm{b}}$ \\ ${ }^{a}$ Institute of Engineering Mechanics, Beijing Jiaotong University, Beijing, 100044, China \\ ${ }^{b}$ School of Mechanical Engineering, Tianjin University, Tianjin, 300350, China
}

${ }^{c}$ School of Civil and Environmental Engineering, University of Technology Sydney, Broadway NSW 2007, Australia

${ }^{d}$ School of Computing, Engineering and Mathematics, Western Sydney University, Penrith, NSW 2751, Australia

\begin{abstract}
.
This paper proposes a new damage index for the crack identification of beams made of functionally graded materials (FGMs) by using the wavelet analysis. The damage index is defined based on the position of the wavelet coefficient modulus maxima in the scale space. The crack is assumed to be an open edge crack and is modeled by a massless rotational spring. It is assumed that the material properties follow exponential distributions along the beam thickness direction. The Timoshenko beam theory is employed to derive the governing equations which are solved analytically to obtain the frequency and mode shape of cracked FGM beams. Then, we apply the continuous wavelet transform (CWT) to the mode shapes of the cracked FGM beams. The locations of the cracks are determined from the sudden changes in the spatial variation of the damage index. An intensity factor, which relates to the size of the crack and the coefficient of the wavelet transform, is employed to estimate the crack depth. The effects of the crack size, the crack location and the Young's modulus ratio on the crack depth detection are investigated.
\end{abstract}

Keywords: Crack identification; Functionally graded materials; Continuous wavelet transform; Damage index

\footnotetext{
*Corresponding author. Tel.: 86-10-51685663; Fax: 86-10-51682094

E-mail address: 11ke@bjtu.edu.cn, 11ke@tju.edu.cn (Liao-Liang Ke)
} 


\section{Introduction}

In recent years, functionally graded materials (FGMs) have found a wide range of applications in many fields [1], such as aerospace, energy, civil engineering, medical, optoelectronics, etc. Their properties are inhomogeneous and change along the spatial location. The gradual change in composition and microstructures of FGMs enables a gradient of properties and performances in the mechanical strength, thermal conductivity, etc. [2] which are highly desirable in many engineering and scientific applications. FGMs possess the ability to reduce the stress/heat concentration, control the deformation and resist the contact damage due to their excellent mechanical performance [3]. However, it is inevitable that small damages will emerge in FGM structures which may become significant damages in severe working environments (vibration, corrosion and high temperature, etc.). It is very important to detect the damage locations and estimate the severity of the damages to prevent potential loss of life and properties. Therefore, the research on damage identification methods for FGM structures has attracted much attention of researchers in recent years.

The occurrence of a crack in a structure reduces the local stiffness and changes the global dynamic responses of the structure. This has led to the substantial development of vibration-based damage identification methods over several decades [4], including the natural frequency-based methods [5-7], the mode shape-based methods [8-10], the modal strain energy-based methods [11-13], etc. Owolabi et al. [14] reported experimental results about the effect of the crack location and depth on the frequency, mode shape and frequency response function of cracked beams. Then two damage identification methods based on changes in the frequency and frequency response function, respectively, were employed to detect cracks for cracked beams. Pandey et al. [15] presented a damage localization method based on the difference between curvature mode shapes of the intact and damaged structures. Appearance of a sharp peak in the curvature mode shapes indicated the presence of damage and its location. Shi et al. [16] proposed a damage detection method based on the change in the modal strain energy. Manoach et al. [17] introduced a new damage index based on Poincaré map of forced vibration and compared their results with popular methods based on modal displacements, modal curvatures and strain energy. Previous studies mainly employed methods that are based on the change of dynamic responses before and after the presence of damage. However, the baseline data from intact structures sometimes are not precise or not available. Furthermore, the natural frequency of a structure is a global parameter, and damage identification methods based on the natural frequency have limitations to determine the location of a local 
damage. On the other hand, the mode shape of a structure is the other modal parameter and contains the local information of the structure. Damage detection method based on the mode shape is quite sensitive to the damage location. Moreover, the mode shape of a structure can be measured accurately by a large number of nodes using the non-contact vibration measurement, such as scanning laser Doppler vibrometer (SLDV) and digital image correlation (DIC), etc. [18-20].

Recently, the wavelet-based damage detection methods have been studied by many researchers. The continuous wavelet transform (CWT) is one of signal processing method to detect singularity. By using CWT, the mode shape of the damaged structure can be employed to detect the damage location and its severity. Wang and Deng [21] conducted numerical studies on the damage identification of structures using CWT. They applied the Haar wavelet and Gabor wavelet to analyze the static and impact responses of cracked beams and plates. Hong et al. [22] proved that wavelet function with at least two vanishing moments should be used to detect damage in beams. They showed the estimation of the crack depth using the Lipschitz exponent of wavelet coefficients. Douka et al. [23] processed the first mode shape of cracked beams by CWT with sym 4 wavelet which has four vanishing moments. They suggested that the wavelet with higher vanishing moment had more advantages for detecting the crack location and depth in beams. This method was utilized for the crack detection of double-cracked beams by Loutridis et al. [24]. Rucka et al. [25] conducted the crack localization in beams using CWT of the static deflection. Zhu and Law [26] studied the operational deflection time history of a bridge subject to a moving load by using CWT. The location of the crack was determined from the location of the minimum of the wavelet coefficient. To resist the effect of measurement noise, Cao et al. [27] proposed an improved curvature mode shape based on the wavelet transform and Teager energy operator for damage detection in multi-cracked beams. Furthermore, after CWT of the finite-length data, such as the static deflection, the mode shape and the response of forced vibration, wavelet coefficients have larger values in the vicinity of the signal edge. The reason for the occurrence of edge effect is that CWT is defined as an infinite integration, and the analysis window of the wavelet extends beyond the length of data [28]. To avoid the edge effect, the extension of the original input data [29-31], the modification of the wavelet functions [32] and the application of window functions [33, 34] were implemented. Most recently, Zhou and Li et al. [35] proposed a damage index based on CWT and mode shape curvatures in composite laminates with cutout. Tao et al. [36] used CWT to process the dynamic response of cracked fiber-metal laminated beams under moving loads for crack detection. 
The aforementioned literatures were related to studies on the damage identification methods for homogeneous and laminated structures. For the application of vibration-based damage identification methods on FGM structures, the first step is to obtain the dynamic responses of the structures. Many researchers conducted the vibration analysis of cracked FGM structures. Ke et al. [37] presented the flexural vibration of a cracked Timoshenko beam made of FGMs. In the vibration analysis, the crack was assumed as the massless spring whose stiffness depended on the stress intensity factors. Wei et al. [38] carried out a free vibration analysis of cracked FGM beams based on transfer matrix method. Rajasekaran et al. [39] studied natural frequencies and mode shapes of bi-directional FGM beams with cracks using the Euler-Bernoulli beam theory and the finite element approach. So far, the reported studies on the damage identification of FGM structures are still quite limited. Yu et al. [40] used the p-version finite element method to calculate vibration characteristics of cracked FGM beams and developed a crack identification method based on the frequency change. Lu et al. [41] presented a sensitivity-based finite element model updating approach to identify the local damages in axially functionally graded beams.

In this study, we propose a new damage index for the crack identification of FGM beams using CWT. Free vibration behaviors of cracked FGM beams with different boundary conditions are studied by using the Timoshenko beam theory and the massless rotational spring model. The fundamental mode shapes of the cracked FGM beams are analyzed by using CWT. To identify the accurate position of a crack and reduce the edge effect, a new damage index is defined according to the position of the wavelet coefficient modulus maxima in the scale space. The merit of this method is that it does not need to compare with results of the intact beam. To estimate damage extent, the intensity factor is calculated by the Lipschitz regulation of the wavelet coefficient. Moreover, the effects of the crack location, the crack depth and the Young's modulus ratio on the intensity factor are discussed in details. These methods are further employed in the analysis of FGM beams with multiple cracks.

\section{Free vibration analysis}

Fig. 1a shows an FGM beam with length $L$ and thickness $h$. There is an open edge crack with crack depth $a$ and located at $L_{1}$ from the left end of the beam. It is assumed that the Young's modulus $E(z)$ and mass density $\rho(z)$ vary in the thickness direction with the exponential function as

$$
E(z)=E_{m} e^{\beta z}, \rho(z)=\rho_{m} e^{\beta z},
$$


where $\beta=\ln \left(E_{1} / E_{2}\right) / h$ is the gradient index, $E_{1}$ and $E_{2}$ are the elastic modulus of top and bottom surfaces of the beam, and $E_{m}$ and $\rho_{m}$ are the Young's modulus and the mass density at the midplane of the beam ( $z=0$ ), respectively. The Poisson's ratio $\mu$ is taken to be a constant.

The massless rotational spring model is used to simulate the edge crack as shown in Fig. 1b. It is assumed that the crack is always open and parallel to the z-axis. The rotational spring connects two separated parts of the beam at the crack location. The bending stiffness $K_{T}$ of the spring can be given as

$$
K_{T}=\frac{1}{G}
$$

where $G$ is the flexibility. At the cracked location, it can be expressed as [42]

$$
\frac{\left(1-\mu^{2}\right) K_{1}^{2}}{E(a)}=\frac{M^{2}}{2} \frac{\mathrm{d} G}{\mathrm{~d} a},
$$

where $M$ is the bending moment at the crack location, $K_{1}$ is the stress intensity factor (SIF) under the mode I bending load, and $E(a)$ is the Young's modulus at the crack tip.

Erdogan and $\mathrm{Wu}$ [43] studied the surface crack problem for a plate with functionally graded properties. They gave the detailed results of the SIFs with various crack depth. Then, we can write the relation between SIF $K_{1}$ and crack depth ratio $\xi$ from the results given by Erdogan and Wu [43]

$$
K_{1}=\frac{6 M \sqrt{\pi h \xi}}{h^{2}} F(\xi), \quad \xi=\frac{a}{h}, \quad \xi \leq 0.7
$$

where $F(\xi)$ is given as

$$
\begin{gathered}
E_{2} / E_{1}=0.2: F(\xi)=1.910-2.752 \xi-4.742 \xi^{2}+146.776 \xi^{3}-770.750 \xi^{4} \\
+1947.830 \xi^{5}-2409.170 \xi^{6}+1177.980 \xi^{7}, \\
E_{2} / E_{1}=1: F(\xi)=1.150-1.662 \xi+21.667 \xi^{2}-192.451 \xi^{3}+909.375 \xi^{4} \\
-2124.310 \xi^{5}+2395.830 \xi^{6}-1031.750 \xi^{7}, \\
E_{2} / E_{1}=5: F(\xi)=0.650-0.859 \xi+12.511 \xi^{2}-72.627 \xi^{3}+267.910 \xi^{4} \\
-535.236 \xi^{5}+545.139 \xi^{6}-211.706 \xi^{7} .
\end{gathered}
$$

The expression of $F(\xi)$ for different Young's modulus ratio can be obtained by using Lagrange interpolation formula. Substitution of Eq. (4) into Eq. (3) leads to 


$$
\mathrm{G}=\int_{0}^{\xi} \frac{72 \pi\left(1-\mu^{2}\right) \xi F^{2}(\xi)}{E(\xi h) h^{2}} \mathrm{~d} \xi .
$$

Compared with the exponential distribution, the power law distribution is used more widely in FGMs. However, the SIF of the edge crack for the power law case is very difficult to analytically obtain by using the conventional method. So far, most of the SIF results of FGMs were for the exponential distribution. This paper uses the exponential distribution for which the SIFs were given by Erdogan and Wu [43]. That is the reason of the exponential model used in the present paper.

Based on the Timoshenko beam theory [44], the kinetic energy $T$ of the cracked FGM beam is given by [37]

$$
\begin{aligned}
& T=\frac{1}{2} \int_{0}^{L_{1}}\left[I_{1}\left(\frac{\partial U_{1}}{\partial t}\right)^{2}+2 I_{2}\left(\frac{\partial U_{1}}{\partial t}\right)\left(\frac{\partial \Psi_{1}}{\partial t}\right)+I_{3}\left(\frac{\partial \Psi_{1}}{\partial t}\right)^{2}+I_{1}\left(\frac{\partial W_{1}}{\partial t}\right)^{2}\right] \mathrm{d} x \\
& +\frac{1}{2} \int_{L_{1}}^{L}\left[I_{1}\left(\frac{\partial U_{2}}{\partial t}\right)^{2}+2 I_{2}\left(\frac{\partial U_{2}}{\partial t}\right)\left(\frac{\partial \Psi_{2}}{\partial t}\right)+I_{3}\left(\frac{\partial \Psi_{2}}{\partial t}\right)^{2}+I_{1}\left(\frac{\partial W_{2}}{\partial t}\right)^{2}\right] \mathrm{d} x
\end{aligned}
$$

and the potential energy $V$ is written as

$$
\begin{aligned}
& V=\frac{1}{2} K_{T}\left[\Psi_{2}\left(L_{1}\right)-\Psi_{1}\left(L_{1}\right)\right]^{2}+\frac{1}{2} \int_{0}^{L_{1}}\left[N_{x 1} \frac{\partial U_{1}}{\partial x}+M_{x 1} \frac{\partial \Psi_{1}}{\partial x}+Q_{x 1}\left(\frac{\partial W_{1}}{\partial x}+\Psi_{1}\right)\right] \mathrm{d} x, \\
& +\frac{1}{2} \int_{L_{1}}^{L}\left[N_{x 2} \frac{\partial U_{2}}{\partial x}+M_{x 2} \frac{\partial \Psi_{2}}{\partial x}+Q_{x 2}\left(\frac{\partial W_{2}}{\partial x}+\Psi_{2}\right)\right] \mathrm{d} x
\end{aligned}
$$

where the subscript $i=1,2$ refers to the left sub-beam and right sub-beam, $U_{i}$ and $W_{i}$ are displacement components at the midplane, $\Psi_{i}$ is the rotation of the cross section of the beam, $N_{x i}, M_{x i}$ and $Q_{x i}$ are the normal resultant force, bending moment and transverse shear force, $x$ is the longitudinal coordinate, and $t$ is time, respectively.

By using the Hamilton principle,

$$
\int_{0}^{t}(\delta T-\delta V) \mathrm{d} t=0
$$

we can derive the governing equations of the cracked FGM beam as

$$
\begin{gathered}
\frac{\partial N_{x i}}{\partial x}=I_{1} \frac{\partial^{2} U_{i}}{\partial t^{2}}+I_{2} \frac{\partial^{2} \Psi_{i}}{\partial t^{2}} \\
\frac{\partial Q_{x i}}{\partial x}=I_{1} \frac{\partial^{2} W_{i}}{\partial t^{2}}
\end{gathered}
$$




$$
\frac{\partial M_{x i}}{\partial x}-Q_{x i}=I_{2} \frac{\partial^{2} U_{i}}{\partial t^{2}}+I_{3} \frac{\partial^{2} \Psi_{i}}{\partial t^{2}}
$$

where $N_{x i}, M_{x i}$ and $Q_{x i}$ are denoted as

$$
\begin{aligned}
& N_{x i}=A_{11} \frac{\partial U_{i}}{\partial x}+B_{11} \frac{\partial \Psi_{i}}{\partial x} \\
& M_{x i}=B_{11} \frac{\partial U_{i}}{\partial x}+D_{11} \frac{\partial \Psi_{i}}{\partial x} \\
& Q_{x i}=\kappa A_{55}\left(\frac{\partial W_{i}}{\partial x}+\Psi_{i}\right)
\end{aligned}
$$

where $\kappa=5 / 6$ is the shear correction factor, $A_{11}, B_{11}, D_{11}$ and $A_{55}$ are stiffness components, and $I_{1}, I_{2}$ and $I_{3}$ are inertia terms, respectively. They are defined by the following formulas.

$$
\begin{aligned}
\left\{A_{11}, B_{11}, D_{11}\right\} & =\int_{-h / 2}^{h / 2} \frac{E(z)}{1-\mu^{2}}\left\{1, z, z^{2}\right\} \mathrm{d} z, \\
A_{55} & =\int_{-h / 2}^{h / 2} \frac{E(z)}{2(1+\mu)} \mathrm{d} z, \\
\left\{I_{1}, I_{2}, I_{3}\right\} & =\int_{-h / 2}^{h / 2} \rho(z)\left\{1, z, z^{2}\right\} \mathrm{d} z .
\end{aligned}
$$

In the present study, three kinds of boundary conditions are considered:

clamped-free (C-F): $x=0, U_{1}=W_{1}=\Psi_{1}=0 ; x=L, \mathrm{~N}_{x 2}=M_{x 2}=Q_{x 2}=0$,

clamped-clamped (C-C): $x=0, U_{1}=W_{1}=\Psi_{1}=0 ; x=L, U_{2}=W_{2}=\Psi_{2}=0$,

hinged-hinged (H-H): $x=0, U_{1}=W_{1}=M_{x 1}=0 ; x=L, U_{2}=W_{2}=M_{x 2}=0$.

At the cracked location $x=L_{1}$, the following compatibility conditions are required:

$$
U_{1}=U_{2}, W_{1}=W_{2}, K_{T}\left(\Psi_{1}-\Psi_{2}\right)=M_{1}, N_{x 1}=N_{x 2}, M_{x 1}=M_{x 2}, Q_{x 1}=Q_{x 2}
$$

By introducing the dimensionless quantities:

$$
\begin{aligned}
& \zeta=\frac{x}{L}, \eta=\frac{L}{h},\left(\bar{U}_{i}, \bar{W}_{i}\right)=\frac{\left(U_{i}, W_{i}\right)}{h}, \bar{K}_{T}=\frac{K_{T}}{A_{110} h}, \omega=\Omega L \sqrt{\frac{I_{10}}{A_{110}}}, \tau=\frac{t}{L} \sqrt{\frac{A_{110}}{I_{10}}}, \bar{\Psi}_{i}=\Psi_{i}, \\
& \left(\bar{I}_{1}, \bar{I}_{2}, \bar{I}_{3}\right)=\left(\frac{I_{1}}{I_{10}}, \frac{I_{2}}{I_{10} h}, \frac{I_{3}}{I_{10} h^{2}}\right),\left(a_{11}, a_{55}, b_{11}, d_{11}\right)=\left(\frac{A_{11}}{A_{110}}, \frac{A_{55}}{A_{110}}, \frac{B_{11}}{A_{110} h}, \frac{D_{11}}{A_{110} h^{2}}\right),
\end{aligned}
$$


we can re-write the dimensionless governing equations as

$$
\begin{gathered}
a_{11} \frac{\partial^{2} \bar{U}_{i}}{\partial \zeta^{2}}+b_{11} \frac{\partial^{2} \bar{\Psi}_{i}}{\partial \zeta^{2}}=\bar{I}_{1} \frac{\partial^{2} \bar{U}_{i}}{\partial \tau^{2}}+\bar{I}_{2} \frac{\partial^{2} \bar{\Psi}_{i}}{\partial \tau^{2}}, \\
\kappa a_{55}\left(\frac{\partial^{2} \bar{W}_{i}}{\partial \zeta^{2}}+\eta \frac{\partial \bar{\Psi}_{i}}{\partial \zeta^{2}}\right)=\bar{I}_{1} \frac{\partial^{2} \bar{W}_{i}}{\partial \tau^{2}}, \\
b_{11} \frac{\partial^{2} \bar{U}_{i}}{\partial \zeta^{2}}+d_{11} \frac{\partial^{2} \bar{\Psi}_{i}}{\partial \zeta^{2}}-\kappa \eta a_{55}\left(\frac{\partial \bar{W}_{i}}{\partial \zeta}+\eta \bar{\Psi}_{i}\right)=\bar{I}_{2} \frac{\partial^{2} \bar{U}_{i}}{\partial \tau^{2}}+\bar{I}_{3} \frac{\partial^{2} \bar{\Psi}_{i}}{\partial \tau^{2}},
\end{gathered}
$$

and the dimensionless boundary conditions as

$$
\begin{gathered}
\bar{U}_{1}=\bar{W}_{1}=\bar{\Psi}_{1}=0, \text { at } \zeta=0, \\
a_{11} \frac{\partial \bar{U}_{2}}{\partial \zeta}+\mathrm{b}_{11} \frac{\partial \bar{\Psi}_{2}}{\partial \zeta}=b_{11} \frac{\partial \bar{U}_{2}}{\partial \zeta}+\mathrm{d}_{11} \frac{\partial \bar{\Psi}_{2}}{\partial \zeta}=\frac{\partial \bar{W}_{2}}{\eta \partial \zeta}+\bar{\Psi}_{2}=0, \text { at } \zeta=1,
\end{gathered}
$$

for the C-F FGM beam,

$$
\begin{aligned}
& \bar{U}_{1}=\bar{W}_{1}=\bar{\Psi}_{1}=0, \text { at } \zeta=0, \\
& \bar{U}_{2}=\bar{W}_{2}=\bar{\Psi}_{2}=0, \text { at } \zeta=1,
\end{aligned}
$$

for the C-C FGM beam,

$$
\begin{gathered}
\bar{U}_{1}=\bar{W}_{1}=b_{11} \frac{\partial \bar{U}_{1}}{\partial \zeta}+\mathrm{d}_{11} \frac{\partial \bar{\Psi}_{1}}{\partial \zeta}=0, \text { at } \zeta=0, \\
\bar{U}_{2}=\bar{W}_{2}=b_{11} \frac{\partial \bar{U}_{2}}{\partial \zeta}+\mathrm{d}_{11} \frac{\partial \bar{\Psi}_{2}}{\partial \zeta}=0, \text { at } \zeta=1,
\end{gathered}
$$

for the H-H FGM beam, and the dimensionless compatibility conditions at the cracked section,

$$
\begin{gathered}
\bar{U}_{1}=\bar{U}_{2}, \bar{W}_{1}=\bar{W}_{2} \\
\bar{K}_{T}\left(\bar{\Psi}_{1}-\bar{\Psi}_{2}\right)=b_{11} \frac{\partial \bar{U}_{1}}{\eta \partial \zeta}+\mathrm{d}_{11} \frac{\partial \bar{\Psi}_{1}}{\eta \partial \zeta}, a_{11} \frac{\partial \bar{U}_{1}}{\partial \zeta}+\mathrm{b}_{11} \frac{\partial \bar{\Psi}_{1}}{\partial \zeta}=a_{11} \frac{\partial \bar{U}_{2}}{\partial \zeta}+\mathrm{b}_{11} \frac{\partial \bar{\Psi}_{2}}{\partial \zeta} \\
b_{11} \frac{\partial \bar{U}_{1}}{\partial \zeta}+\mathrm{d}_{11} \frac{\partial \bar{\Psi}_{1}}{\partial \zeta}=b_{11} \frac{\partial \bar{U}_{2}}{\partial \zeta}+\mathrm{d}_{11} \frac{\partial \bar{\Psi}_{2}}{\partial \zeta}, \frac{\partial \bar{W}_{1}}{\eta \partial \zeta}+\bar{\Psi}_{1}=\frac{\partial \bar{W}_{2}}{\eta \partial \zeta}+\bar{\Psi}_{2}
\end{gathered}
$$

For the harmonic vibration of the beam, the displacements and rotation are assumed to be

$$
\begin{aligned}
& \bar{U}_{i}(\zeta, \tau)=u_{i}(\zeta) e^{\mathrm{i} \omega \tau}, \\
& \bar{W}_{i}(\zeta, \tau)=w_{i}(\zeta) e^{\mathrm{i} \omega \tau},
\end{aligned}
$$




$$
\bar{\Psi}_{i}(\zeta, \tau)=\psi_{i}(\zeta) e^{\mathrm{i} \omega \tau}
$$

Neglecting the axial inertia and substituting Eqs. (30)-(32) into Eqs. (20)-(22) lead to

$$
\begin{gathered}
a_{11} \frac{\mathrm{d}^{2} u_{i}}{\mathrm{~d} \zeta^{2}}+b_{11} \frac{\mathrm{d}^{2} \psi_{i}}{\mathrm{~d} \zeta^{2}}=-\bar{I}_{2} \omega^{2} \psi_{i}, \\
\kappa a_{55}\left(\frac{\mathrm{d}^{2} w_{i}}{\mathrm{~d} \zeta^{2}}+\eta \frac{\mathrm{d} \psi_{i}}{\mathrm{~d} \zeta}\right)=-\bar{I}_{1} \omega^{2} w_{i}, \\
b_{11} \frac{\mathrm{d}^{2} u_{i}}{\mathrm{~d} \zeta^{2}}+d_{11} \frac{\mathrm{d}^{2} \psi_{i}}{\mathrm{~d} \zeta^{2}}-\kappa \eta a_{55}\left(\frac{\mathrm{d} w_{i}}{\mathrm{~d} \zeta}+\eta \psi_{i}\right)=-\bar{I}_{3} \omega^{2} \psi_{i}
\end{gathered}
$$

Eliminating $u_{i}$ from Eqs. (33)-(35), they are decoupled into two independent equations

$$
\begin{aligned}
& \frac{\mathrm{d}^{4} w_{i}}{\mathrm{~d} \zeta^{4}}+(m+n) \frac{\mathrm{d}^{2} w_{i}}{\mathrm{~d} \zeta^{2}}+(m n-p) w_{i}=0 \\
& \frac{\mathrm{d}^{4} \psi_{i}}{\mathrm{~d} \zeta^{4}}+(m+n) \frac{\mathrm{d}^{2} \psi_{i}}{\mathrm{~d} \zeta^{2}}+(m n-p) \psi_{i}=0
\end{aligned}
$$

where

$$
D=d_{11}-\frac{b_{11}^{2}}{a_{11}}, \quad m=\frac{\bar{I}_{1}}{\kappa a_{55}}, \quad n=\frac{1}{D}\left(\bar{I}_{3} \omega^{2}-\frac{b_{11} \bar{I}_{2} \omega^{2}}{a_{11}}\right), \quad p=\frac{\bar{I}_{1} \eta^{2} \omega^{2}}{D}
$$

Since $(m-n)^{2}+4 p>0$, the transverse displacement, axial displacement and rotation can be solved as

$$
\begin{gathered}
u_{i}(\zeta)=f_{i 1} q_{3} \sinh \left(k_{1} \zeta\right)+f_{i 2} q_{3} \cosh \left(k_{1} \zeta\right)-f_{i 3} q_{4} \sin \left(k_{2} \zeta\right)+f_{i 4} q_{4} \cos \left(k_{2} \zeta\right)+g_{i} \zeta+r_{i}, \\
w_{i}(\zeta)=f_{i 1} \cosh \left(k_{1} \zeta\right)+f_{i 2} \sinh \left(k_{1} \zeta\right)+f_{i 3} \cos \left(k_{2} \zeta\right)+f_{i 4} \sin \left(k_{2} \zeta\right), \\
\psi_{i}(\zeta)=f_{i 1} q_{1} \sinh \left(k_{1} \zeta\right)+f_{i 2} q_{1} \cosh \left(k_{1} \zeta\right)-f_{i 3} q_{2} \sin \left(k_{2} \zeta\right)+f_{i 4} q_{2} \sin \left(k_{2} \zeta\right) .
\end{gathered}
$$

where

$$
\begin{gathered}
k_{1}=\sqrt{\sqrt{\left(\frac{m-n}{2}\right)^{2}+p}-\frac{m+n}{2}}, \quad k_{2}=\sqrt{\sqrt{\left(\frac{m-n}{2}\right)^{2}+p}+\frac{m+n}{2}} \\
q_{1}=-\frac{k_{1}^{2}+m}{k_{1} \eta}, \quad q_{1}=-\frac{\left(k_{2}^{2}-m\right)}{k_{2} \eta}, q_{3}=-\frac{q_{1}}{k_{1}^{2}}\left(\frac{b_{11} k_{1}^{2}}{a_{11}}+\frac{\bar{I}_{2} \omega^{2}}{a_{11}}\right), \quad q_{4}=-\frac{q_{1}}{k_{2}^{2}}\left(\frac{b_{11} k_{2}^{2}}{a_{11}}-\frac{\bar{I}_{2} \omega^{2}}{a_{11}}\right) .
\end{gathered}
$$

The unknown constants $f_{1 j}, f_{2 j}, g_{i}$ and $r_{i}(i=1,2 ; j=1,2,3,4)$ are determined from the boundary conditions and compatibility conditions in Eqs. (23)-(29). Then, applying these boundary conditions and compatibility conditions of the $\mathrm{C}-\mathrm{F}, \mathrm{C}-\mathrm{C}$ and $\mathrm{H}-\mathrm{H}$ beams, we can obtain the nonlinear algebraic equations in the matrix form 


$$
[H(\omega)]\{X\}=\{0\}
$$

where $\{X\}=\left\{f_{11}, f_{12}, f_{13}, f_{14}, g_{1}, r_{1}, \ldots, f_{i 1}, f_{i 2}, f_{i 3}, f_{i 4}, g_{i}, r_{i}\right\}^{T} ;[H(\omega)]$ is a matrix nonlinearly dependent on the natural frequency. According to the condition of the equations with the nonzero solution, the determinant of $H(\omega)$ must satisfy

$$
\operatorname{det}[H(\omega)]=0
$$

The determinants $H_{C-F}, H_{C-C}, H_{H-H}$ for C-F, C-C, H-H FGM beams are given in Appendix A, respectively. According to Eqs. (43) and (44), the natural frequencies and the corresponding mode shapes can be obtained.

\section{Crack identification using continuous wavelet transform}

The wavelet transform is a valid mathematical tool for analyzing singularities and irregular structures in signals. So it can characterize the local singularities from the mode shape of cracked beams. This damage detection technique was applied in homogenous and laminated structures successfully.

\subsection{Continuous wavelet transform}

In CWT technique, we introduce that the function $\varphi(x) \in L^{2}(R)$ is said to be a wavelet if and only if its Fourier transform $\hat{\varphi}(\varpi)$ satisfies [45]

$$
\int_{-\infty}^{+\infty} \frac{|\hat{\varphi}(\varpi)|^{2}}{|\varpi|} \mathrm{d} \varpi=C_{\varphi}<+\infty
$$

Eq. (45) implies $\varphi(x)$ with a zero average, i.e.,

$$
\int_{-\infty}^{+\infty} \varphi(x) \mathrm{d} x=0
$$

CWT of a signal function $f(x) \in L^{2}(R)$ is defined by

$$
W f(b, s)=f * \frac{1}{\sqrt{s}} \tilde{\varphi}\left(\frac{b}{s}\right)=f * \tilde{\varphi}_{s}(b)=\int_{-\infty}^{+\infty} f(x) \tilde{\varphi}_{b, s} \mathrm{~d} x
$$

where $b$ and $s$ are the translation parameter and scale parameter, respectively, $W f(b, s)$ is the wavelet transform coefficient of $f(x)$ at the bounded scale interval, $\tilde{\varphi}(x)$ denotes the complex conjugate of 
the wavelet function $\varphi(x)$, the symbol $(*)$ means the convolution of two functions, and $\tilde{\varphi}_{b, s}=\frac{1}{\sqrt{s}} \tilde{\varphi}\left(\frac{x-b}{s}\right)$

The type of singularities is often measured with the Lipschitz exponent $\alpha$. It is defined that a function $f(x)$ has Lipschitz exponent $\alpha \geq 0$ at $x=v$, if there exists $K>0$ that is independent of $v$, and a polynomial $p_{v}$ of degree $\chi$ ( $\chi$ is the largest integer satisfying $\chi \leq \alpha$ ). Therefore, we have [46]

$$
\begin{gathered}
f(x)=p_{v}(x)+\varepsilon_{v}(x), \\
\left|\varepsilon_{v}(x)\right| \leq K|x-v|^{\alpha} .
\end{gathered}
$$

For example, if $f(x)$ is $\chi$ times continuously differentiable in a neighborhood of $v$, then $p_{v}$ is the Taylor expansion of $f(x)$ at $v$. Lipschitz exponent $\alpha$ is equal to $\chi$. In contrast, when a function is not differentiable at $v$, Lipschitz exponent $\alpha \in(0,1)$ while $p_{v}(x)$ is equal to $f(v)$.

To measure Lipschitz exponent $\alpha$ by ignoring polynomial $p_{v}$ in Eq. (47), we must impose that the wavelet has enough number of vanishing moments $n_{v}$. A wavelet $\varphi(x)$ is said to have $n_{v}$ vanishing moments, if and only if the positive integer $k<n_{v}$, it satisfies

$$
\int_{-\infty}^{+\infty} x^{k} \varphi(x) \mathrm{d} x=0
$$

It is clear that the wavelet having $n_{v}$ vanishing moments is orthogonal to polynomials of up to degree $n_{v}-1 . W f(b, s)$ can be reduced to

$$
W f(b, s)=\frac{1}{\sqrt{s}} \int_{-\infty}^{+\infty}\left(p_{v}(x)+\varepsilon_{v}(x)\right) \varphi\left(\frac{x-b}{s}\right) \mathrm{d} x=W \varepsilon_{v}(b, s),
$$

In other words, Lipschitz exponent measured from $W \varepsilon_{v}(b, s)$ is $\alpha \leq n_{v}$.

Mallat [45] proved that if $\varphi(x)$ has $n_{v}$ vanishing moments, we can introduce a fast decay function $\vartheta(x)$ to satisfy Eq. (45). It is defined by 


$$
\varphi(x)=(-1)^{p_{v}} \frac{\mathrm{d}^{p_{v}} \vartheta(x)}{\mathrm{d} x^{p_{v}}},
$$

where integer $p_{v}<n_{v}$. Hence $W f(b, s)$ is written as

$$
W f(b, s)=f * \frac{1}{\sqrt{s}} \tilde{\varphi}\left(\frac{b}{s}\right)=\frac{s^{p_{v}}}{\sqrt{s}} f * \frac{\mathrm{d}^{p_{v}} \tilde{\vartheta}(b / s)}{\mathrm{d} b^{p_{v}}}=s^{p_{v}} \frac{\mathrm{d}^{p_{v}}}{\mathrm{~d} b^{p_{v}}}\left(f * \tilde{\vartheta}_{s}\right)(b)
$$

where $\tilde{\vartheta}_{s}(b)=s^{-1 / 2} \vartheta(-b / s)$.

Eq. (53) is called the multi-scale differential operator that builds the relationship between the decay of $|W f(b, s)|$ when the scale $s$ decreases and the differentiability of $f(x)$. Singularities are detected where the value of $|W f(b, s)|$ decays as the scale $s$ decreases to zero. At the fine scale $s_{0}$, the singularities including the discontinuity and non-differentiability create the wavelet coefficient modulus maxima at the location of singularities, i.e.,

$$
\frac{\partial W f\left(b_{0}, s_{0}\right)}{\partial b}=0
$$

The line, which is composed of the modulus maxima in the scale space $(b, s)$, is called the maxima line.

The Lipschitz regularity at $b=b_{0}$ can be calculated from the decay of the maxima modulus $\left|W f\left(b_{0}, s\right)\right|$ with the decrease of scale $s$. According to Jaffard [47], if $f(x) \in L^{2}(R)$ has the Lipschitz exponent $\alpha \leq n_{v}$ at $b=b_{0}$, then the asymptotic behavior of $|W f(b, s)|$ near $b=b_{0}$ becomes

$$
|W f(b, s)| \leq A s^{\alpha+\frac{1}{2}}\left(1+\left|\frac{b-b_{0}}{s}\right|^{\alpha}\right)
$$

and when $b=b_{0}$, Eq. (55) can be reduced to

$$
|W f(b, s)| \leq A s^{\alpha+\frac{1}{2}}
$$

which can be further written as

$$
\log _{2}|W f(b, s)| \leq \log _{2}|A|+\left(\alpha+\frac{1}{2}\right) \log _{2} s
$$

where Lipschitz regularity at $b_{0}$ is the maximum slope of $\log _{2}|W f(b, s)|$ as a function of $\log _{2} s$ along the maxima line converging to $b_{0}$, and $\log _{2}|A|$ is the intercept of the function of $\log _{2} s$. 
Assuming that $W f\left(b_{0}, s\right)$ is the wavelet transform coefficient at the maxima line of the largest slope at the location $b_{0}$, inequality (57) is rewritten as [24]

$$
\log _{2}\left|W f\left(b_{0}, s\right)\right|=\log _{2}|A|+\left(\alpha+\frac{1}{2}\right) \log _{2} s .
$$

Then, Lipschitz exponent $\alpha$ and constant $A$ which characterize the Lipschitz regulation of the wavelet coefficient at location $b_{0}$ can be estimated by the least squares method.

\subsection{Crack identification method using CWT}

In order to identify the damage of a cracked FGM beam, it is assumed that the function $f(x)$ is the mode shape of the beam and $\varphi(x)$ is a real wavelet. $W f(b, s)$ is the coefficient of the wavelet transform of the mode shape. At the crack location, the wavelet transform coefficient change suddenly in the spatial variation. Moreover, the decay of the wavelet coefficient modulus maxima converges to the crack location with decreasing scale.

For the beam with an open edge crack, the crack location is always a maxima point when the scale parameter varies. The maxima line of the largest slope is approximately perpendicular to abscissa in the scale space $(b, s)$. In this paper, a new damage index is defined by

$$
\mathrm{DI}(x)=\sum_{s=s_{\min }}^{s_{\max }} D W f, D W f= \begin{cases}0 & \frac{\partial W f(x, s)}{\partial b} \neq 0, \\ 1 & \frac{\partial W f(x, s)}{\partial b}=0\end{cases}
$$

where $x$ denotes the distance of the detection point at the beam length direction from the left end, $s_{\min }$ is the minimum scale parameter, and $s_{\max }$ is the maximum scale parameter.

In fact, the maximum damage index is located at the crack location where maxima lines converge to. Then, for the damage severity estimation, the Lipschitz exponent $\alpha$ and the constant $A$ can be measured by both the wavelet modulus of maxima lines of the largest slope and Eq. (58). If the crack depth is changed, Lipschitz exponent has the fixed value; however, the constant $A$ varies with the crack depth. So, the constant $A$ can be defined as the intensity factor to characterize the depth of crack.

The compatibility conditions (18) and (29) show that the mode shape of the cracked FGM beam is a 
second order non-differentiable function at the crack location. Hence, the Lipschitz exponent is between 1 and 2, i.e.,

$$
1<\alpha<2 \text {. }
$$

Therefore, the number of vanishing moments of the wavelet should be chosen at least 2 . It is noted that wavelet with the higher vanishing moments and smaller support width can provide more stable performance. Hence, we can choose wavelet family with higher vanishing moments but relatively short support width. After some numerical tests, the sym8 wavelet is suitable to the crack identification in this paper.

\section{Numerical results and discussion}

Table 1 gives the fundamental frequency of an isotropic homogeneous cantilever beam with an open edge crack. The parameters used in this example are: $L=0.2 \mathrm{~m}, h=0.0078 \mathrm{~m}, E=216 \mathrm{GPa}$, $\rho=7850 \mathrm{~kg} / \mathrm{m}^{3}, \mu=0.28$, and $a / h=0.2,0.4$. The finite element results given by Kisa et al. [48] is also given in Table 1. Obviously, both results show a good agreement.

By using the wavelet analysis, this section will study the crack identification of C-F, H-H and C-C FGM beams containing one or two open edge cracks. Unless otherwise stated, it is assumed that the beam thickness $h=0.1 \mathrm{~m}$ and the slenderness ratio $L / h=6$. The ratio of Young's modulus is taken as $E_{2} / E_{1}=5,1,0.2$. The top surface of the FGM beam is $100 \%$ aluminum with material parameters $E_{1}=70 \mathrm{GPa}$ and $\rho_{1}=2780 \mathrm{~kg} / \mathrm{m}^{3}$. The Poisson's ratio $\mu$ is taken to be constant value 0.33 .

It should be pointed out that the axial inertia is omitted in Eqs. (33)-(35) in order to solve the problem analytically. In our previous paper [49], we solved the cracked FGM Timoshenko beam by using the Ritz method where the axial inertia was taken into account. To address the error induced by omitting the axial inertia, Table 2 gives the comparison between the Ritz results and present results of the cracked FGM Timoshenko beam. The parameters in this example are selected as $E_{1}=70 \mathrm{GPa}, \rho_{1}=2780 \mathrm{~kg} / \mathrm{m}^{3}$, $\mu=0.33, L / h=6, h=0.1 \mathrm{~m}, a / h=0.2$ and $L_{1} / L=0.5$. The data in the brackets are the relative error between the Ritz results with axial inertia and present results without axial inertia. It can be seen that the error between the Ritz results and present results is small. This indicates that the omission of the axial inertia will have a slight effect on the crack identification of FGM beams. 


\subsection{Mode analysis}

Fig. 2 presents the effect of the crack depth ratio $a / h$ on the first mode shape of the FGM beam with $E_{2} / E_{1}=5$ and $L_{1} / L=0.5$. The mode shape for the intact FGM beam is also plotted for comparison. Deflection data with 501 sampling points are normalized so that the maximum deflection value equals one. As can be seen from Fig. 2, the crack has a slight effect on the mode shape of C-F, H-H and C-C FGM beams. We can observe that the mode shapes of the cracked FGM beams and intact FGM beams are nearly the same. Therefore, it is difficult to detect the accurate crack location and distinguish the crack depth according to mode shapes directly. We need to explore a new method for the crack identification of FGM beams. The wavelet transform method, which is one of signal processing methods, has been widely used to detect damage. By using wavelet analysis, the mode shape data are transformed into the spatial domain with multiple scales to provide wavelet coefficients. The sharp data transitions enable wavelet coefficients to create large amplitude, and therefore it can be used for the damage identification of FGM beams. In the next sub-sections, we will give a detailed discussion for the wavelet-based crack identification for FGM beams.

\subsection{Crack location identification}

To identify the crack location, the mode shape of beams is analyzed by using the sym 8 wavelet to obtain wavelet coefficients. The scale $s$ is taken from 1 to 32. Fig. 3 shows the results of the wavelet coefficient modulus $|W f(b, s)|$ for edge cracked FGM beams with $E_{2} / E_{1}=5, L_{1} / L=0.5$ and $a / h=0.3$. For C-C and $\mathrm{H}-\mathrm{H}$ beams, $|W f(b, s)|$ has obvious wavelet coefficient modulus maxima near $x / L=0.5$. The amplitude of $|W f(b, s)|$ decays asymptotically with decreasing scale and converges at $x / L=0.5$. It is proved that the crack location can be indicated by the fluctuations of wavelet coefficients. For a C-F beam, there is no significant perturbation at the crack location. In other words, the wavelet coefficient modulus does not provide the visual information about the crack location. The reason is that the sudden variability of the signal due to crack has a relatively negligible amplitude in comparison to the higher deflection value at free-end. The results of the wavelet transform for C-C and $\mathrm{H}-\mathrm{H}$ beams also have the similar border distortion problem, though they can indicate the presence and location of the crack.

Note that CWT is defined as an infinite integration in Eq. (47). Hence, the mode shape of the finite 
beam must result in the appearance of the higher value at support ends. There is no known method to remove the higher value at support ends. However, the reduction of the edge effect can be implemented by the extrapolated displacement method and modification of CWT. In the present study, we develop a new damage index based on position of the wavelet coefficient maximum at the scale space. It is capable of reducing the edge effect and detecting the location of crack. As mentioned above, the damage index of each sampling point $(\zeta \in[0,1])$ is defined by Eq. (59). The scale parameter $s_{\min }$ and $s_{\max }$ are taken to be 1 and 32, respectively. According to the calculated wavelet coefficient, the position of the wavelet coefficient maximum at scale space can be obtained.

Fig. 4 gives the damage index for FGM beams with an open edge crack and $E_{2} / E_{1}=5$, $L_{1} / L=0.5$ and $a / h=0.3$. It is clearly shown in Fig. 4 that the damage index has a peak at $x / L=0.5$ for all three beams. In fact, the spatial position of the damage index peak indicates the crack location where the wavelet coefficient modulus converges. The value of the damage index around boundaries does not lead to the confusion during the crack detection. The introduction of the damage index can reduce the edge effect compared to the wavelet coefficient modulus. Indeed, the damage index is better than the wavelet coefficient modulus for the crack location identification. Furthermore, the presence of cracks and their locations are well predicted by the damage index for C-C, H-H and C-F FGM beams. The crack localization can be achieved by finding the maximum damage index.

\subsection{Crack depth estimation}

After the crack localization, the damage severity can also be estimated by using the wavelet analysis. The detection of crack depth is implemented by computing the Lipschitz regularity of wavelet maximum lines of the largest slope at the crack position. The intensity factor relating to the wavelet modulus maximum and crack depth can be measured by Eq. (58).

Fig. 5 shows the wavelet modulus maxima lines of the largest slope versus the scale of cracked FGM beams with $E_{2} / E_{1}=5$ and $L_{1} / L=0.5$. The optimized scale $s$ is taken from 4 to 24 . For C-C, H-H and C-F beams, there are a set of parallel lines for different crack depths. By computing the slopes of the lines, the Lipschitz exponents can be solved and are approximately equal to 1.01. This means that the mode shape is the second order non-differentiable at the crack location. The value of $|W f(b, s)|$ becomes larger 
as the crack depth increases. The crack depth can be distinguished by each line's ordinate intercept, which is equal to $\log _{2}|A|$. Therefore, the constant $A$ can be defined as the intensity factor for the crack depth estimation.

Fig. 6 presents the effect of Young's modulus ratio $E_{2} / E_{1}$ on the intensity factor versus crack depth with $L_{1} / L=0.5$. For C-C, H-H and C-F FGM beams, the intensity factor increases as the crack depth increases. When the crack location and depth are fixed, the intensity factor becomes smaller as $E_{2} / E_{1}$ increases. This is due to the fact that the mode shape of FGM beams with lower bending stiffness is more sensitive to crack [37].

Similarly, the mode shape of FGM beams is sensitive to the crack location, which in turn affects the intensity factor. Fig. 7 gives the effect of Young's modulus ratio on the intensity factor versus crack position with $a / h=0.2$. It is observed that the relationship between the crack location and intensity factor is completely different for C-C, H-H and C-F beams. For C-C beams, the intensity factor curve has the turning points at $0.2 \mathrm{~L}, 0.5 \mathrm{~L}$ and $0.8 \mathrm{~L}$. However, the turning point is at $0.5 \mathrm{~L}$ for $\mathrm{H}-\mathrm{H}$ beams and C-F beams do not have the turning point. For a C-F beam, the intensity factor is quite small when the crack is close to the free end. The reduction of the intensity factor is observed when Young's modulus ratio changes from 0.2 to 5.0 .

In summary, the values of the intensity factor are influenced by the Young's modulus ratio, the crack location and the crack depth. Tables 3, 4 and 5 show the intensity factor of C-C, H-H and C-F FGM beams with a single crack, respectively. These detailed data could serve as the referencing value to estimate the depth of crack in future measurements.

\subsection{Double-cracked FGM beam}

Fig. 8 highlights the damage index of the FGM beam with two edge cracks located at $0.2 \mathrm{~L}$ and $0.8 L$ and $E_{2} / E_{1}=5$. The two cracks have the depth ratio $a_{1} / h=0.3$ and $a_{2} / h=0.2$, respectively. The cracks are detected accurately by the position of the peak value, i.e. the No.101 and No.401 sampling points $(0.2 L$ and $0.8 L)$. The crack depth is detected by the Lipschitz regularity of wavelet maximum lines of the largest slope at the crack position. The magnitude of the intensity factor is shown in Table 6 . These results indicate that the present crack identification method can be employed universally for multi-cracked 
beams.

\subsection{The effect of the noise}

In the practical condition, the measurement noise always exists in the displacement mode shape. The performance of crack identification under noisy conditions need to be investigated. For simulating noise, white noise is added to analytical mode shape of the cracked FGM beam. The mode shapes incorporating noise can be obtained by the following formula [26]:

$$
w_{\text {noise }}=w_{\text {calulated }}+Z_{\text {noise }} N_{\text {noise }} \sigma\left(w_{\text {calulated }}\right) \text {, }
$$

where $w_{\text {noise }}$ is the polluted mode shape, $Z_{\text {noise }}$ is the noise level, $N_{\text {oise }}$ is the standard normal distribution vector with a zero mean value and a unit standard deviation, $w_{\text {calulated }}$ is the normalized deflection data, and $\sigma\left(w_{\text {calulated }}\right)$ is the standard deviation for the mode shape.

Fig. 9(a)-(c) shows the damage index DI of the H-H cracked FGM beam $\left(E_{2} / E_{1}=5\right.$, $\left.L_{1} / L=0.5, a_{1} / h=0.3\right)$ with noise level $Z_{\text {noise }}=0.1 \%, 0.5 \%$ and $1 \%$, respectively. The scale parameter $s$ for CWT is taken from 1 to 64 . As expected, the value of the damage index reduces with the increase of the noise level. It is clear that the damage index has a singular peak at $x / L=0.5$ for all three different noise level. Hence, in suitable noisy condition, the presence of cracks and their locations still can be well indicated by the damage index.

\section{Conclusion}

This paper proposes a new damage index for the crack identification of FGM beams. The damage index is defined according to the position of the wavelet coefficient modulus maxima in the scale space. The mode shape of the cracked FGM beam is analyzed by using CWT. The damage index is employed to identify the accurate position of the crack and reduce the edge effect. To estimate damage extent, the intensity factor is calculated by the Lipschitz regulation of the wavelet coefficient.

The results show that:

(1) It is difficult to detect the accurate crack location and distinguish the crack depth according to mode shapes directly;

(2) The results of the wavelet transform for C-C and H-H FGM beams also have the similar edge effect 
problem, though they can still indicate the presence and location of the crack;

(3) The introduction of the damage index can reduce the edge effect compared to the wavelet coefficient modulus. The presence of cracks and their locations are well predicted by the damage index for C-C, H-H and C-F FGM beams. The crack localization can be achieved by finding the maximum damage index; and

(4) The intensity factor is effective for the crack depth estimation of cracked FGM beams. The intensity factor is sensitive to the Young's modulus ratio, the crack location and the crack depth.

\section{Acknowledgements}

The work described in this paper is supported by the Fundamental Research Funds for the Central Universities (Grant No. 2018YJS100) and National Natural Science Foundation of China (Grant No. 11725207).

\section{References}

[1] Naebe M, Shirvanimoghaddam K. Functionally graded materials: A review of fabrication and properties. Applied Materials Today 2016; 5: 223-245.

[2] Koizumi M. FGM activities in Japan. Composites Part B: Engineering 1997; 28: 1-4.

[3] Gupta A, Talha M. Recent development in modeling and analysis of functionally graded materials and structures. Progress in Aerospace Sciences 2015; 79: 1-14.

[4] Fan W and Qiao P. Vibration-based damage identification methods: a review and comparative study. Struct Health Monit 2011; 10: 83-111.

[5] Salawu OS. Detection of structural damage through changes in frequency: a review. Eng Struct 1997; 19: 718-723.

[6] Lee J. Identification of multiple cracks in a beam using natural frequencies. J Sound Vib 2009; $320: 482-490$.

[7] Zhang K, Yan X. Multi-cracks identification method for cantilever beam structure with variable cross-sections based on measured natural frequency changes. J Sound Vib 2017; 387: 53-65.

[8] Whalen TM. The behavior of higher order mode shape derivatives in damaged, beam-like structures. J Sound Vib 2008; 309: 426-464.

[9] Roy K, Ray-Chaudhuri S. Fundamental mode shape and its derivatives in structural damage 
localization. J Sound Vib 2013; 332: 5584-5593.

[10] Huang T, Chaves-Vargas M, Yang J, Schröder K-U. A baseline-free structural damage indicator based on node displacement of structural mode shapes. J Sound Vib 2018; 433: 366-384.

[11] Fan W, Qiao P. A strain energy-based damage severity correction factor method for damage identification in plate-type structures. Mech Syst Sig Process 2012; 28: 660-678.

[12] Zhang J, Guo SL Wu ZS, Zhang QQ. Structural identification and damage detection through long-gauge strain measurements. Eng Struct 2015; 99: 173-183.

[13] Cui H, Xu X, Peng W, Zhou Z, Hong M. A damage detection method based on strain modes for structures under ambient excitation. Measurement 2018; 125: 438-446.

[14] Owolabi GM, Swamidas ASJ, Seshadri R. Crack detection in beams using changes in frequencies and amplitudes of frequency response functions. J Sound Vib 2003; 265: 1-22.

[15] Pandey AK, Biswas M, Samman MM, Damage detection from changes in curvature mode shapes. J Sound Vib 1991; 145:321-332.

[16] Shi ZY, Law SS, Zhang LM. Structural damage detection from modal strain energy change. J Eng Mech 2000; 126: 1216-1223.

[17] Manoach E, Warminski J, Kloda L, Teter A. Numerical and experimental studies on vibration based methods for detection of damage in composite beams. Compos Struct 2017; 170:26-39.

[18] Baqersad J, Poozesh P, Niezrecki C, Avitabile P. Photogrammetry and optical methods in structural dynamics - A review. Mech Syst Sig Process 2017; 86: 17-34.

[19] Reu PL, Rohe DP, Jacobs LD. Comparison of DIC and LDV for practical vibration and modal measurements. Mech Syst Sig Process 2017; 86: 2-16.

[20] Chen DM, Xu YF, Zhu WD. Non-model-based multiple damage identification of beams by a continuously scanning laser Doppler vibrometer system. Measurement 2018;115: 185-196.

[21] Wang Q, Deng X. Damage detection with spatial wavelets. Int J Solids Struct 1999; 36: 3443-3468.

[22] Hong JC, Kim YY, Lee HC, Lee YW. Damage detection using the Lipschitz exponent estimated by the wavelet transform: applications to vibration modes of a beam. Int J Solids Struct 2002; 39:1803-1816.

[23] Douka E, Loutridis S, Trochidis A. Crack identification in beams using wavelet analysis. Int J Solids Struct 2003; 40:3557-3569.

[24] Loutridis S, Douka E, Trochidis A. Crack identification in double-cracked beams using wavelet 
analysis, J Sound Vib 2004; 277:1025-1039.

[25] Rucka M, Wilde K. Crack identification using wavelets on experimental static deflection profiles. Eng Struct 2006; 28:279-288.

[26] Zhu XQ, Law SS. Wavelet-based crack identification of bridge beam from operational deflection time history. Int J Solids Struct 2006; 43:2299-2317.

[27] Cao M, Radzieński M, Xu W, Ostachowicz W. Identification of multiple damage in beams based on robust curvature mode shapes. Mech Syst Sig Process 2014; 46:468-480.

[28] Kijewski T, Ksreem A. On the presence of end effects and their melioration in wavelet-based analysis. J Sound Vib 2002; 256:980-988.

[29] Solís M, Algaba M, Galvín P. Continuous wavelet analysis of mode shapes differences for damage detection. Mech Syst Sig Process 2013; 40:645-666.

[30] Cao M and Qiao P. Integrated wavelet transform and its application to vibration mode shapes for the damage detection of beam-type structures. Smart Mater Struct 2008; 17(5):055014.

[31] Shahsavari V, Chouinard L, Bastien J. Wavelet-based analysis of mode shapes for statistical detection and localization of damage in beams using likelihood ratio test. Eng Struct 2017; 132:494-507.

[32] Boltežar M, Slavič J. Enhancements to the continuous wavelet transform for damping identifications on short signals. Mech Syst Sig Process 2004; 18(5):1065-1076.

[33] Gentile A, Messina A. On the continuous wavelet transforms applied to discrete vibrational data for detecting open cracks in damaged beams. Int J Solids Struct 2003; 40(2):295-315.

[34] Mardasi AG, Wu N, Wu C. Experimental study on the crack detection with optimized spatial wavelet analysis and windowing. Mech Syst Sig Process 2018; 104:619-630.

[35] Zhou J, Li Z, Chen J. Damage identification method based on continuous wavelet transform and mode shapes for composite laminates with cutouts. Compos Struct 2018; 191:12-23.

[36] Tao C, Fu Y, Dai T. Dynamic analysis for cracked fiber-metal laminated beams carrying moving loads and its application for wavelet based crack detection. Compos Struct 2017; 159:463-470.

[37] Ke LL, Yang J, Kitipornchai S, Xiang Y. Flexural vibration and elastic buckling of a cracked Timoshenko beam made of functionally graded materials. Mech Adv Mater Struct; 2009, 16:488-502.

[38] Wei D, Liu YH, Xiang ZH. An analytical method for free vibration analysis of functionally graded beams with edge cracks. J Sound Vib 2012; 331: 1686-1700.

[39] Rajasekaran S, Khaniki HB. Free vibration analysis of bi-directional functionally graded 
single/multi-cracked beams. Int J Mech Sci 2018; 144:341-356.

[40] Yu Z, Chu F. Identification of crack in functionally graded material beams using the p-version of finite element method. J Sound Vib 2009; 325:69-84.

[41] Lu ZR, Lin XX, Chen YM, Huang M. Hybrid sensitivity matrix for damage identification in axially functionally graded beams. Appl Math Model 2017; 41:604-617.

[42] Broek D. Elementary Engineering Fracture Mechanics. Dordrecht: Martinus Nijhoff Publishers 1986.

[43] Erdogan F, Wu BH. The Surface Crack Problem for a Plate With Functionally Graded Properties. ASME. J Appl Mech 1997; 64:449-456.

[44] Reddy JN. Mechanics of Laminated Composite Plates. New York: CRC Press 1997.

[45] Mallat S, Hwang W L. Singularity detection and processing with wavelets. IEEE T Informa Theory $1992 ; 38: 617-643$.

[46] Mallat S. A Wavelet Tour of Signal Processing (Second Edition), San Diego: Academic Press 1999.

[47] Jaffard S. Pointwise smoothness, two-microlocalization and wavelet coefficients. Publ Mat 1991; 35: $155-168$

[48] Kisa M, Brandon J, Topcu M. Free vibration analysis of cracked beams by a combination of finite elements and component mode synthesis methods. Comput Struct 1998; 67:215-223.

[49] Kitipornchaia S, Ke LL, Yang J, Xiang Y. Nonlinear vibration of edge cracked functionally graded Timoshenko beams. J Sound Vib 2009; 324:962-982. 


\section{Appendix A}

Suppose that $\theta_{10}=\cosh \left(k_{1}\right), \theta_{20}=\sinh \left(k_{2}\right), \theta_{30}=\cosh \left(k_{2}\right), \quad \theta_{40}=\sin \left(k_{2}\right)$, $\theta_{11}=\cosh \left(k_{1} \zeta_{0}\right), \theta_{21}=\sinh \left(k_{1} \zeta_{0}\right), \theta_{31}=\cos \left(k_{2} \zeta_{0}\right), \theta_{41}=\sin \left(k_{2} \zeta_{0}\right), \quad K_{T}^{*}=K_{T} / A_{110} h$, $D_{0}=\left(a_{11} q_{3}+b_{11} q_{1}\right) k_{1}, \quad D_{1}=\left(a_{11} q_{4}+b_{11} q_{2}\right) k_{2}, \quad D_{3}=\left(b_{11} q_{3}+d_{11} q_{1}\right) k_{1}, \quad D_{4}=\left(b_{11} q_{4}+d_{11} q_{2}\right) k_{2}$, $J_{0}=k_{1}+\eta q_{1}, J_{1}=k_{2}+\eta q_{2}, \quad J_{3}=\eta K_{T}^{*} q_{1}, J_{4}=\eta K_{T}^{*} q_{2}$.

The determinants $H_{C-F}, H_{C-C}, H_{H-H}$ for C-F, C-C, H-H FGM beams with an edge crack are given as follows, respectively.

$H_{C-F}=\left|\begin{array}{cccccccccccc}0 & q_{3} & 0 & q_{4} & 0 & 1 & 0 & 0 & 0 & 0 & 0 & 0 \\ 1 & 0 & 1 & 0 & 0 & 0 & 0 & 0 & 0 & 0 & 0 & 0 \\ 0 & q_{1} & 0 & q_{2} & 0 & 0 & 0 & 0 & 0 & 0 & 0 & 0 \\ 0 & 0 & 0 & 0 & 0 & 0 & D_{0} \theta_{10} & D_{0} \theta_{20} & -D_{1} \theta_{30} & -D_{1} \theta_{40} & a_{11} & 0 \\ 0 & 0 & 0 & 0 & 0 & 0 & D_{3} \theta_{10} & D_{3} \theta_{20} & -D_{4} \theta_{30} & -D_{4} \theta_{40} & b_{11} & 0 \\ 0 & 0 & 0 & 0 & 0 & 0 & J_{0} \theta_{20} & J_{0} \theta_{10} & -J_{1} \theta_{40} & J_{1} \theta_{30} & 0 & 0 \\ q_{3} \theta_{21} & q_{3} \theta_{11} & -q_{4} \theta_{41} & q_{4} \theta_{31} & \zeta_{0} & 1 & -q_{3} \theta_{21} & -q_{3} \theta_{11} & q_{4} \theta_{41} & -q_{4} \theta_{31} & -\zeta_{0} & -1 \\ \theta_{11} & \theta_{21} & \theta_{31} & \theta_{41} & 0 & 0 & -\theta_{11} & -\theta_{21} & -\theta_{31} & -\theta_{41} & 0 & 0 \\ D_{0} \theta_{11} & D_{0} \theta_{21} & -D_{1} \theta_{31} & -D_{1} \theta_{41} & a_{11} & 0 & -D_{0} \theta_{11} & -D_{0} \theta_{21} & D_{1} \theta_{31} & D_{1} \theta_{41} & -a_{11} & 0 \\ D_{3} \theta_{11} & D_{3} \theta_{21} & -D_{4} \theta_{31} & -D_{4} \theta_{41} & b_{11} & 0 & -D_{3} \theta_{11} & -D_{3} \theta_{21} & D_{4} \theta_{31} & D_{4} \theta_{41} & -b_{11} & 0 \\ J_{0} \theta_{21} & J_{0} \theta_{11} & -J_{1} \theta_{41} & J_{1} \theta_{31} & 0 & 0 & -J_{0} \theta_{21} & J_{0} \theta_{11} & J_{1} \theta_{41} & -J_{1} \theta_{31} & 0 & 0 \\ J_{3} \theta_{21}+D_{3} \theta_{11} & J_{3} \theta_{21}+D_{3} \theta_{21} & -J_{4} \theta_{41}-D_{4} \theta_{31} & J_{4} \theta_{31}-D_{4} \theta_{41} & b_{11} & 0 & -J_{3} \theta_{21} & -J_{3} \theta_{11} & J_{4} \theta_{41} & -J_{4} \theta_{31} & 0 & 0\end{array}\right|$




$$
H_{C-C}=\left|\begin{array}{cccccccccccc}
0 & q_{3} & 0 & q_{4} & 0 & 1 & 0 & 0 & 0 & 0 & 0 & 0 \\
1 & 0 & 1 & 0 & 0 & 0 & 0 & 0 & 0 & 0 & 0 & 0 \\
0 & q_{1} & 0 & q_{2} & 0 & 0 & 0 & 0 & 0 & 0 & 0 & 0 \\
0 & 0 & 0 & 0 & 0 & 0 & q_{3} \theta_{20} & q_{3} \theta_{10} & -q_{4} \theta_{40} & q_{4} \theta_{30} & 1 & 1 \\
0 & 0 & 0 & 0 & 0 & 0 & \theta_{10} & \theta_{20} & \theta_{30} & \theta_{40} & 0 & 0 \\
0 & 0 & 0 & 0 & 0 & 0 & q_{1} \theta_{20} & q_{1} \theta_{10} & -q_{2} \theta_{40} & q_{2} \theta_{30} & 0 & 0 \\
q_{3} \theta_{21} & q_{3} \theta_{11} & -q_{4} \theta_{41} & q_{4} \theta_{31} & \zeta_{0} & 1 & -q_{3} \theta_{21} & -q_{3} \theta_{11} & q_{4} \theta_{41} & -q_{4} \theta_{31} & -\zeta_{0} & -1 \\
\theta_{11} & \theta_{21} & \theta_{31} & \theta_{41} & 0 & 0 & -\theta_{11} & -\theta_{21} & -\theta_{31} & -\theta_{41} & 0 & 0 \\
D_{0} \theta_{11} & D_{0} \theta_{21} & -D_{1} \theta_{31} & -D_{1} \theta_{41} & a_{11} & 0 & -D_{0} \theta_{11} & -D_{0} \theta_{21} & D_{1} \theta_{31} & D_{1} \theta_{41} & -a_{11} & 0 \\
D_{3} \theta_{11} & D_{3} \theta_{21} & -D_{4} \theta_{31} & -D_{4} \theta_{41} & b_{11} & 0 & -D_{3} \theta_{11} & -D_{3} \theta_{21} & D_{4} \theta_{31} & D_{4} \theta_{41} & -b_{11} & 0 \\
J_{0} \theta_{21} & J_{0} \theta_{11} & -J_{1} \theta_{41} & J_{1} \theta_{31} & 0 & 0 & -J_{0} \theta_{21} & J_{0} \theta_{11} & J_{1} \theta_{41} & -J_{1} \theta_{31} & 0 & 0 \\
J_{3} \theta_{21}+D_{3} \theta_{11} & J_{3} \theta_{21}+D_{3} \theta_{21} & -J_{4} \theta_{41}-D_{4} \theta_{31} & J_{4} \theta_{31}-D_{4} \theta_{41} & b_{11} & 0 & -J_{3} \theta_{21} & -J_{3} \theta_{11} & J_{4} \theta_{41} & -J_{4} \theta_{31} & 0 & 0
\end{array}\right|
$$




\section{Figure captions}

Fig.1. A cracked FGM beam (a) and the massless rotational spring model connecting two sub-beams (b).

Fig.2. Effect of crack depth ratio on the first normalized mode shape of the FGM beam with $E_{2} / E_{1}=5$ and $L_{1} / L=0.5$ : (a) C-C beam; (b) H-H beam; (c) C-F beam.

Fig.3. Wavelet coefficients modulus for edge cracked FGM beams with $E_{2} / E_{1}=5, L_{1} / L=0.5$ and $a / h=0.3$ for scale $s=1 \sim 32:$ (a) C-C beam; (b) H-H beam; (c) C-F beam.

Fig.4. Damage index for FGM beams with an open edge crack and $E_{2} / E_{1}=5, L_{1} / L=0.5$ and $a / h=0.3:$ (a) C-C beam; (b) H-H beam; (c) C-F beam.

Fig.5. Wavelet modulus maxima of edge cracked FGM beams with $E_{2} / E_{1}=5$ and $L_{1} / L=0.5$ : (a) C-C beam; (b) H-H beam; (c) C-F beam.

Fig.6. Effect of the Young's modulus ratio on the intensity factor of edge cracked FGM beams with $L_{1} / L=0.5$ versus crack depth: (a) C-C beam; (b) H-H beam; (c) C-F beam.

Fig.7. Effect of the Young's modulus ratio on the intensity factor of edge cracked FGM beams with $a / h=0.2$ versus crack position: (a) C-C beam; (b) H-H beam; (c) C-F beam.

Fig.8. Damage index of the FGM beam with two edge cracks $\left(E_{2} / E_{1}=5, L_{1} / L=0.2, L_{2} / L=0.8\right.$, $a_{1} / h=0.3, a_{2} / h=0.2$ ): (a) C-C beam; (b) H-H beam; (c) C-F beam.

Fig. 9 Damage index of the $\mathrm{H}-\mathrm{H}$ cracked FGM beam with single crack $\left(E_{2} / E_{1}=5, L_{1} / L=0.5\right.$, $a_{1} / h=0.3$ ) in noisy conditions: (a) $Z_{\text {noise }}=0.1 \%$; (b) $Z_{\text {noise }}=0.5 \%$; (c) $Z_{\text {noise }}=1 \%$. 
Table 1 Fundamental frequency of an isotropic homogenous cantilever beam with an edge crack.

\begin{tabular}{|c|c|c|c|c|c|c|c|c|}
\hline & \multicolumn{2}{|c|}{$L_{1} / L=0.2$} & \multicolumn{2}{|c|}{$L_{1} / L=0.4$} & \multicolumn{2}{|c|}{$L_{1} / L=0.6$} & \multicolumn{2}{|c|}{$L_{1} / L=0.8$} \\
\hline & Present & $\begin{array}{l}\text { Kisa et } \\
\text { al. [48] }\end{array}$ & Present & $\begin{array}{l}\text { Kisa et } \\
\text { al. [48] }\end{array}$ & Present & $\begin{array}{l}\text { Kisa et } \\
\text { al. [48] }\end{array}$ & Present & $\begin{array}{l}\text { Kisa et } \\
\text { al. [48] }\end{array}$ \\
\hline 0.2 & 1020.103 & 1020.137 & 1029.871 & 1030.095 & 1034.963 & 1035.284 & 1036.534 & 1036.884 \\
\hline 0.4 & 967.7161 & 966.9525 & 1006.995 & 1006.856 & 1029.033 & 1029.262 & 1036.070 & 1036.414 \\
\hline
\end{tabular}


Table 2 The effect of axial inertia on the fundamental frequency of cracked FGM beams.

\begin{tabular}{|c|c|c|c|c|}
\hline \multirow[t]{3}{*}{$E_{2} / E_{1}$} & \multicolumn{2}{|l|}{$\mathrm{H}-\mathrm{H}$} & \multicolumn{2}{|l|}{ C-C } \\
\hline & with axial & \multirow{2}{*}{$\begin{array}{c}\text { without axial } \\
\text { inertia }\end{array}$} & with axial & \multirow{2}{*}{$\begin{array}{c}\text { without axial } \\
\text { inertia }\end{array}$} \\
\hline & inertia [49] & & inertia [49] & \\
\hline 0.2 & 0.41921 & $0.41415(1.22 \%)$ & 0.82329 & $0.81249(1.32 \%)$ \\
\hline 1 & 0.42454 & $0.41987(1.11 \%)$ & 0.86882 & $0.85841(1.21 \%)$ \\
\hline 5.0 & 0.43757 & $0.43232(1.21 \%)$ & 0.83964 & $0.82894(1.29 \%)$ \\
\hline
\end{tabular}


Table 3 Intensity factor of cracked C-C FGM beam $\left(10^{-4}\right)$

\begin{tabular}{ccccccc}
\hline & & \multicolumn{5}{c}{$a / h$} \\
$L_{1} / L$ & $E_{2} / E_{1}$ & 0.1 & 0.2 & 0.3 & 0.4 & 0.5 \\
\cline { 3 - 6 } & 5 & 0.121 & 0.571 & 1.179 & 1.814 & 2.421 \\
\hline 0.1 & 1 & 0.185 & 0.812 & 1.547 & 2.289 & 2.932 \\
& 0.2 & 0.259 & 1.045 & 1.904 & 2.654 & 3.237 \\
& 5 & 0.034 & 0.128 & 0.263 & 0.431 & 0.621 \\
0.2 & 1 & 0.051 & 0.180 & 0.360 & 0.580 & 0.814 \\
& 0.2 & 0.064 & 0.253 & 0.478 & 0.723 & 0.940 \\
0.3 & 5 & 0.105 & 0.373 & 0.779 & 1.314 & 1.959 \\
& 1 & 0.151 & 0.532 & 1.094 & 1.838 & 2.694 \\
& 0.2 & 0.190 & 0.679 & 1.401 & 2.253 & 3.124 \\
& 5 & 0.194 & 0.690 & 1.410 & 2.284 & 3.219 \\
& 1 & 0.280 & 0.977 & 1.935 & 3.054 & 4.121 \\
& 0.2 & 0.353 & 1.235 & 2.413 & 3.591 & 4.593 \\
& 5 & 0.222 & 0.777 & 1.544 & 2.433 & 3.361 \\
& 1 & 0.319 & 1.088 & 2.083 & 3.200 & 4.272 \\
& 0.2 & 0.402 & 1.362 & 2.563 & 3.736 & 4.735 \\
\hline
\end{tabular}


Table 4 Intensity factor of cracked H-H FGM beam $\left(10^{-4}\right)$

\begin{tabular}{|c|c|c|c|c|c|c|}
\hline \multirow{2}{*}{$L_{1} / L$} & \multirow{2}{*}{$E_{2} / E_{1}$} & \multicolumn{5}{|c|}{$a / h$} \\
\hline & & 0.1 & 0.2 & 0.3 & 0.4 & 0.5 \\
\hline \multirow[t]{3}{*}{0.1} & 5 & 0.052 & 0.151 & 0.430 & 0.888 & 1.709 \\
\hline & 1 & 0.067 & 0.208 & 0.603 & 1.327 & 3.781 \\
\hline & 0.2 & 0.055 & 0.343 & 0.966 & 3.502 & 5.593 \\
\hline \multirow[t]{3}{*}{0.2} & 5 & 0.101 & 0.391 & 0.894 & 1.644 & 2.708 \\
\hline & 1 & 0.133 & 0.510 & 1.151 & 2.142 & 3.527 \\
\hline & 0.2 & 0.182 & 0.764 & 1.773 & 3.249 & 5.098 \\
\hline \multirow[t]{3}{*}{0.3} & 5 & 0.152 & 0.555 & 1.179 & 2.013 & 3.011 \\
\hline & 1 & 0.196 & 0.707 & 1.477 & 2.505 & 3.638 \\
\hline & 0.2 & 0.279 & 1.024 & 2.144 & 3.441 & 4.560 \\
\hline \multirow[t]{3}{*}{0.4} & 5 & 0.176 & 0.627 & 1.275 & 2.057 & 2.882 \\
\hline & 1 & 0.226 & 0.790 & 1.569 & 2.469 & 3.367 \\
\hline & 0.2 & 0.320 & 1.118 & 2.171 & 3.207 & 4.123 \\
\hline \multirow[t]{3}{*}{0.5} & 5 & 0.177 & 0.646 & 1.276 & 2.020 & 2.806 \\
\hline & 1 & 0.238 & 0.810 & 1.553 & 2.414 & 3.271 \\
\hline & 0.2 & 0.334 & 1.119 & 2.127 & 3.125 & 3.990 \\
\hline
\end{tabular}


Table 5 Intensity factor of cracked C-F FGM beam $\left(10^{-5}\right)$

\begin{tabular}{|c|c|c|c|c|c|c|}
\hline \multirow{2}{*}{$L_{1} / L$} & \multirow{2}{*}{$E_{2} / E_{1}$} & \multicolumn{5}{|c|}{$a / h$} \\
\hline & & 0.1 & 0.2 & 0.3 & 0.4 & 0.5 \\
\hline \multirow[t]{3}{*}{0.1} & 5 & 0.481 & 1.829 & 3.582 & 5.630 & 7.797 \\
\hline & 1 & 0.754 & 2.566 & 4.819 & 7.302 & 9.657 \\
\hline & 0.2 & 0.965 & 3.166 & 5.930 & 8.680 & 11.070 \\
\hline \multirow[t]{3}{*}{0.2} & 5 & 0.415 & 1.520 & 3.136 & 5.149 & 7.443 \\
\hline & 1 & 0.624 & 2.198 & 4.342 & 6.909 & 9.570 \\
\hline & 0.2 & 0.769 & 2.743 & 5.455 & 8.431 & 11.285 \\
\hline \multirow[t]{3}{*}{0.3} & 5 & 0.339 & 1.273 & 2.693 & 4.586 & 6.925 \\
\hline & 1 & 0.514 & 1.853 & 3.812 & 6.359 & 9.262 \\
\hline & 0.2 & 0.631 & 2.347 & 4.891 & 7.988 & 11.302 \\
\hline \multirow[t]{3}{*}{0.4} & 5 & 0.257 & 1.011 & 2.208 & 3.901 & 6.139 \\
\hline & 1 & 0.391 & 1.497 & 3.182 & 5.580 & 8.598 \\
\hline & 0.2 & 0.498 & 1.902 & 4.170 & 7.230 & 10.924 \\
\hline \multirow[t]{3}{*}{0.5} & 5 & 0.186 & 0.754 & 1.695 & 3.081 & 5.065 \\
\hline & 1 & 0.285 & 1.127 & 2.487 & 4.548 & 7.446 \\
\hline & 0.2 & 0.354 & 1.451 & 3.316 & 6.096 & 9.935 \\
\hline \multirow[t]{3}{*}{0.6} & 5 & 0.123 & 0.512 & 1.178 & 2.200 & 3.759 \\
\hline & 1 & 0.194 & 0.773 & 1.755 & 3.347 & 5.777 \\
\hline & 0.2 & 0.237 & 1.003 & 2.386 & 4.612 & 8.100 \\
\hline \multirow[t]{3}{*}{0.7} & 5 & 0.052 & 0.300 & 0.667 & 1.334 & 2.358 \\
\hline & 1 & 0.106 & 0.423 & 1.033 & 2.071 & 3.749 \\
\hline & 0.2 & 0.135 & 0.562 & 1.450 & 2.924 & 5.497 \\
\hline \multirow[t]{3}{*}{0.8} & 5 & 0.006 & 0.133 & 0.336 & 0.583 & 1.057 \\
\hline & 1 & 0.012 & 0.207 & 0.452 & 0.931 & 2.017 \\
\hline & 0.2 & 0.028 & 0.278 & 0.645 & 1.614 & 2.928 \\
\hline
\end{tabular}


Table 6 Intensity factor of the FGM beam with two cracks $\left(10^{-4}\right)$

\begin{tabular}{ccc}
\hline & $I F_{1}$ & $I F_{2}$ \\
\hline C-C & 0.258 & 0.136 \\
H-H & 0.866 & 0.338 \\
C-F & 0.313 & 0.0101 \\
\hline
\end{tabular}




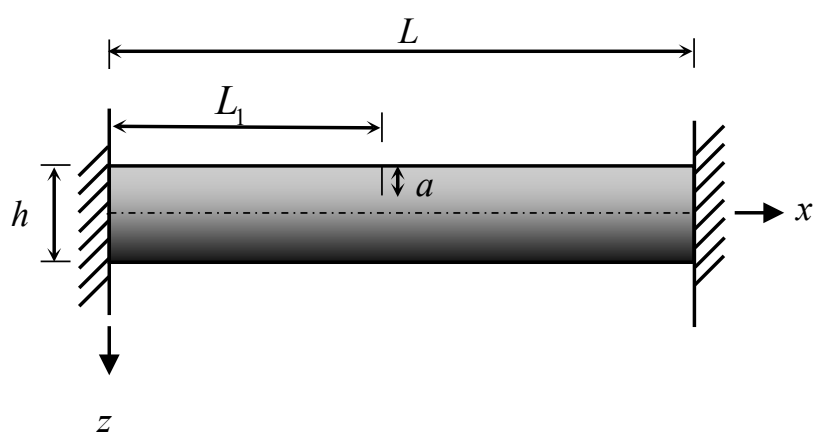

(a)

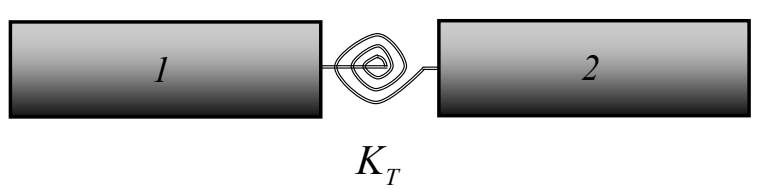

(b)

Fig.1. A cracked FGM beam (a) and the massless rotational spring model connecting two sub-beams (b). 


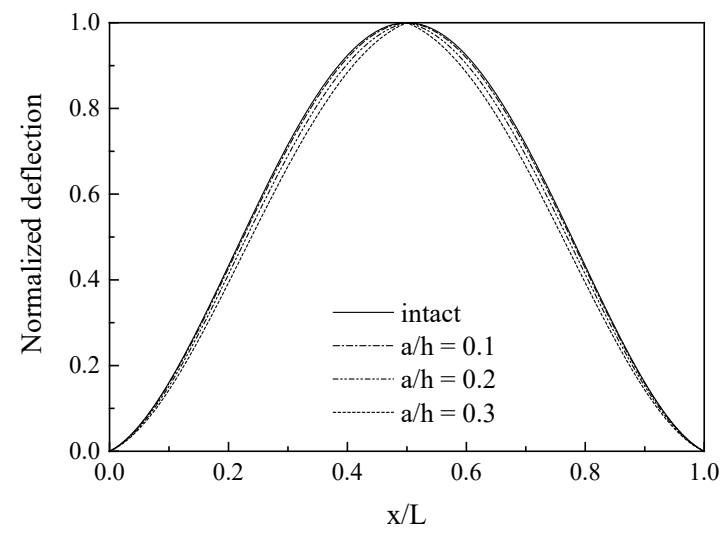

(a)

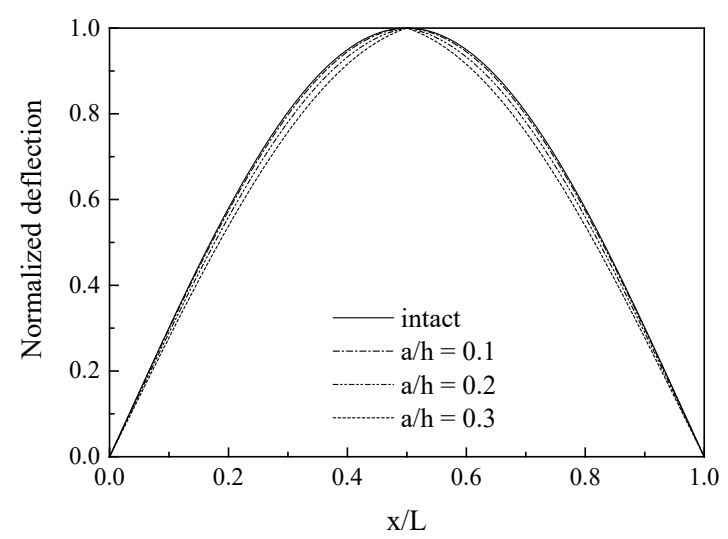

(b)

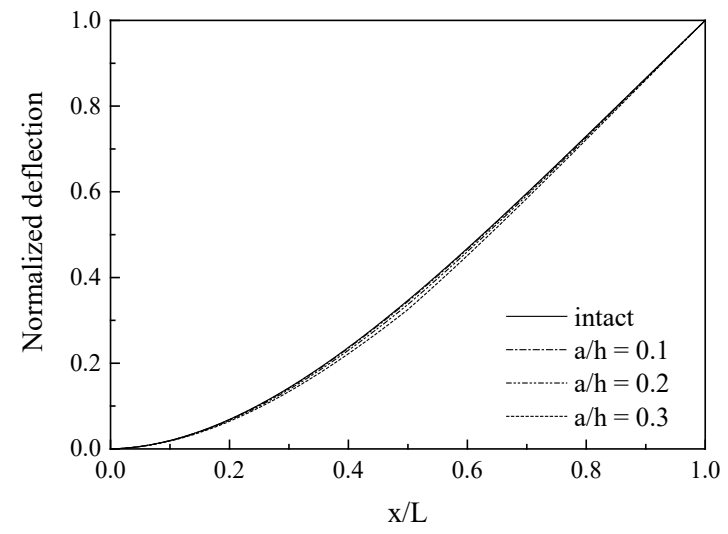

(c)

Fig.2. Effect of crack depth ratio on the first normalized mode shape of the FGM beam with $E_{2} / E_{1}=5$ and $L_{1} / L=0.5$ : (a) C-C beam; (b) H-H beam; (c) C-F beam. 


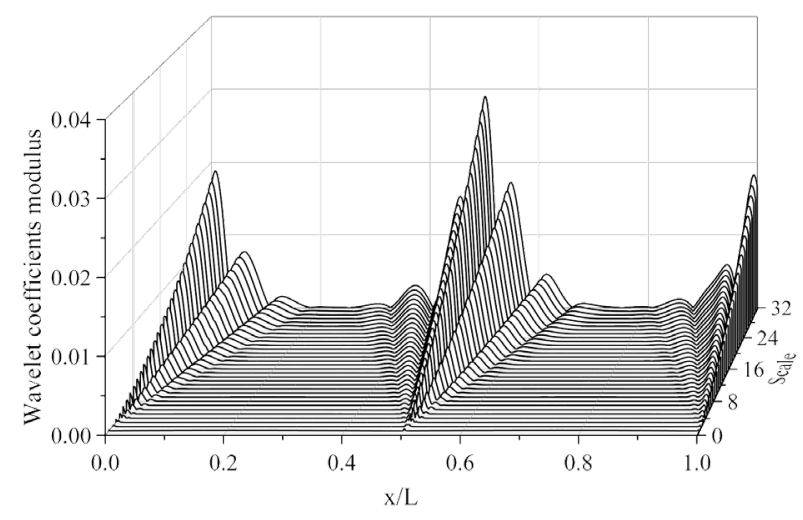

(a)

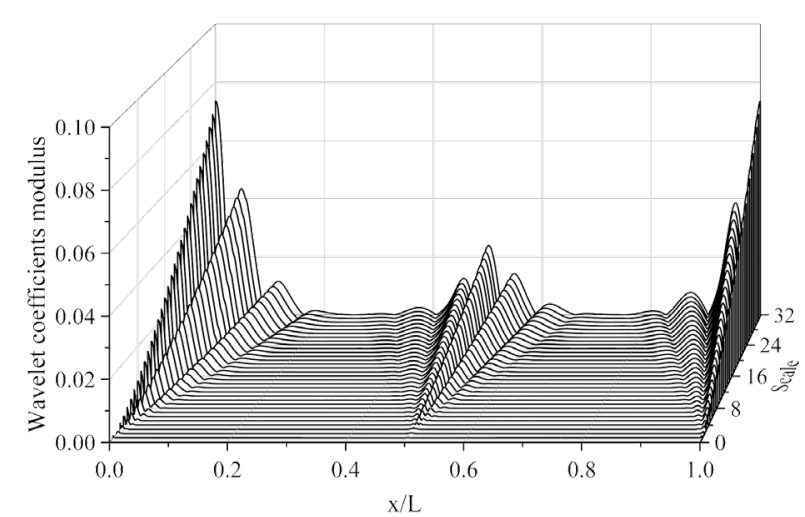

(b)

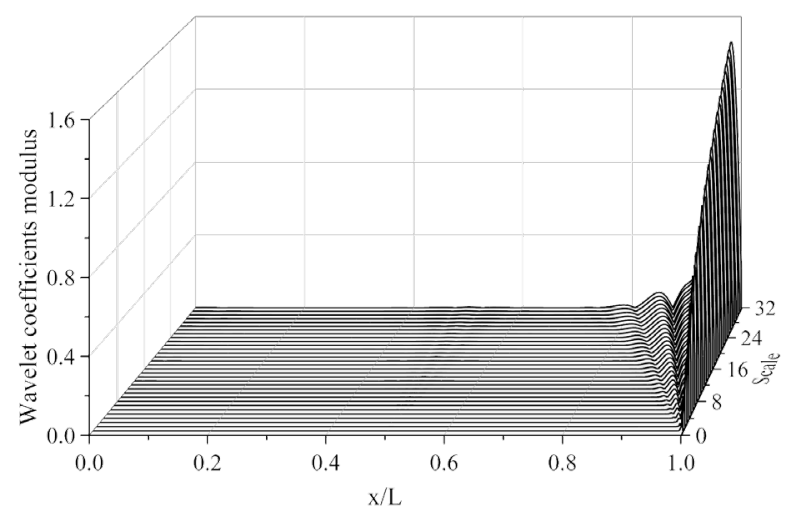

(c)

Fig.3. Wavelet coefficients modulus for edge cracked FGM beams with $E_{2} / E_{1}=5, L_{1} / L=0.5$ and $a / h=0.3$ for scale $s=1 \sim 32:$ (a) C-C beam; (b) H-H beam; (c) C-F beam. 


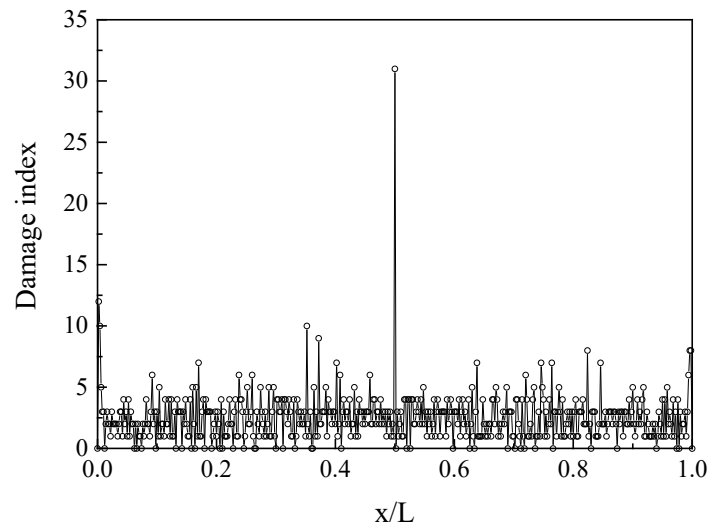

(a)

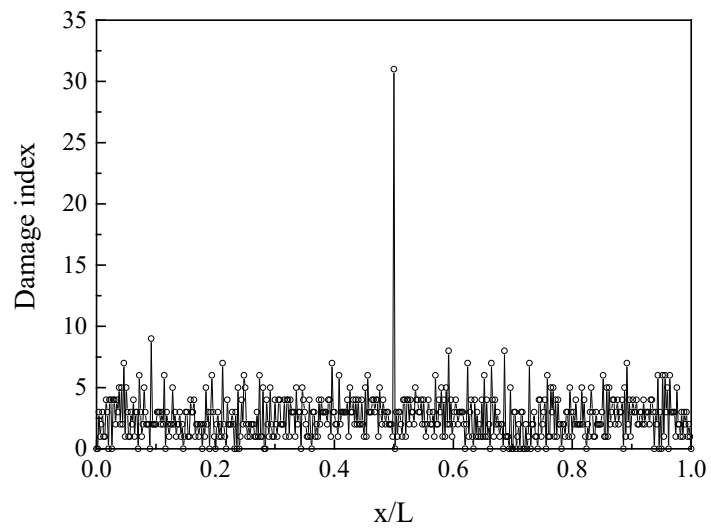

(b)

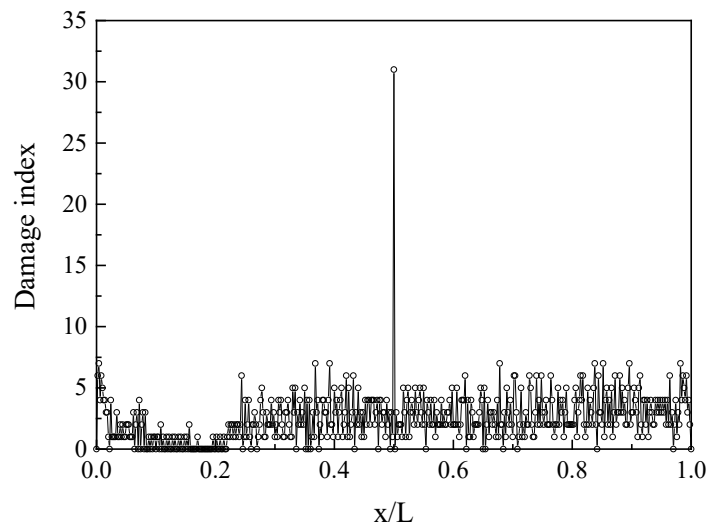

(c)

Fig.4. Damage index for FGM beams with an open edge crack and $E_{2} / E_{1}=5, L_{1} / L=0.5$ and $a / h=0.3:$ (a) C-C beam; (b) H-H beam; (c) C-F beam. 


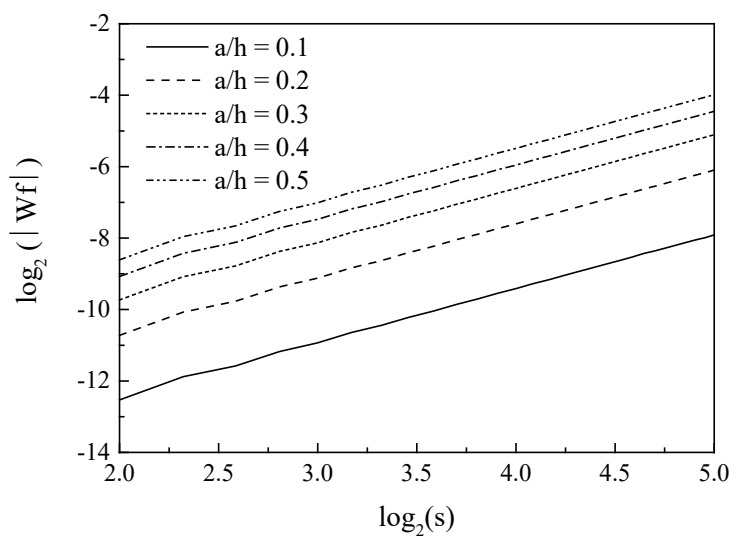

(a)

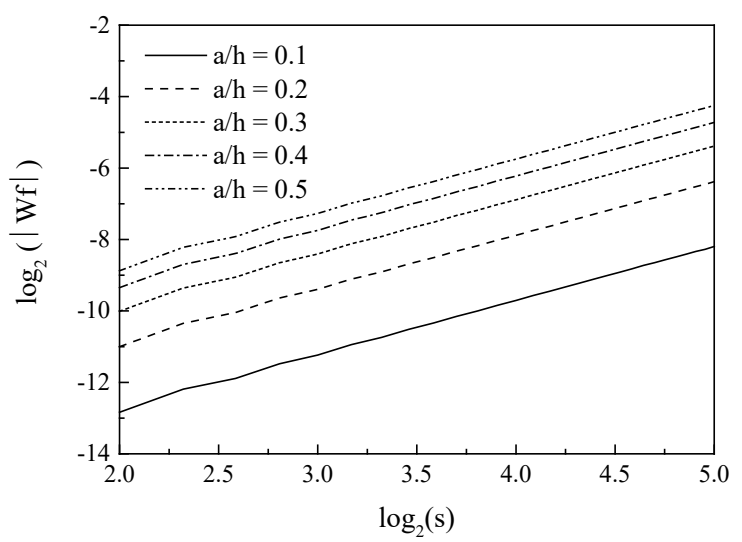

(b)

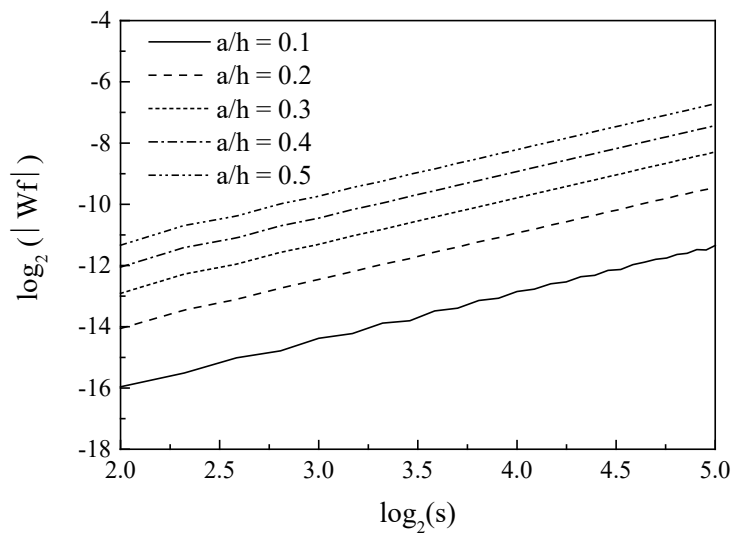

(c)

Fig.5. Wavelet modulus maxima of edge cracked FGM beams with $E_{2} / E_{1}=5$ and $L_{1} / L=0.5$ : (a)

C-C beam; (b) H-H beam; (c) C-F beam. 


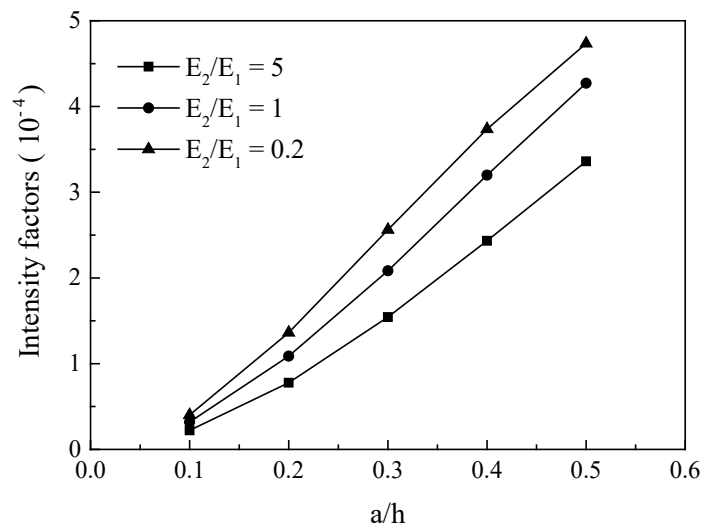

(a)

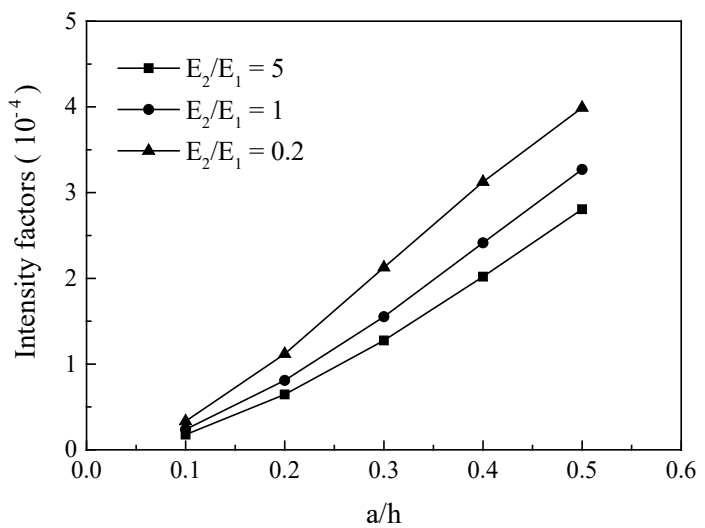

(b)

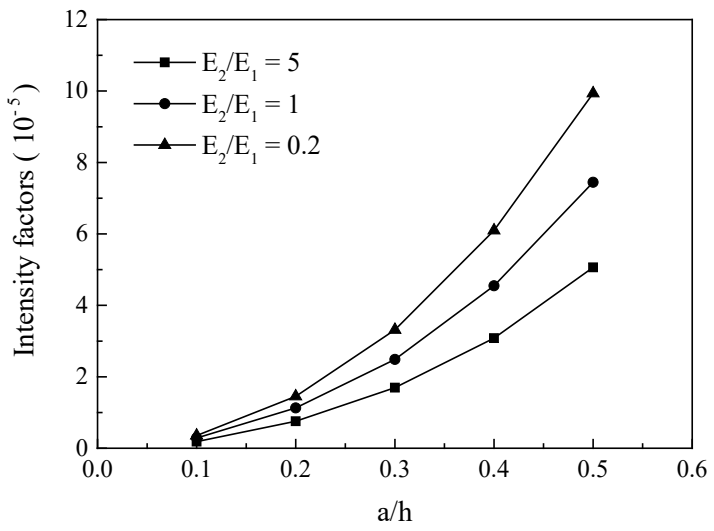

(c)

Fig.6. Effect of the Young's modulus ratio on the intensity factor of edge cracked FGM beams with $L_{1} / L=0.5$ versus crack depth: (a) C-C beam; (b) H-H beam; (c) C-F beam. 


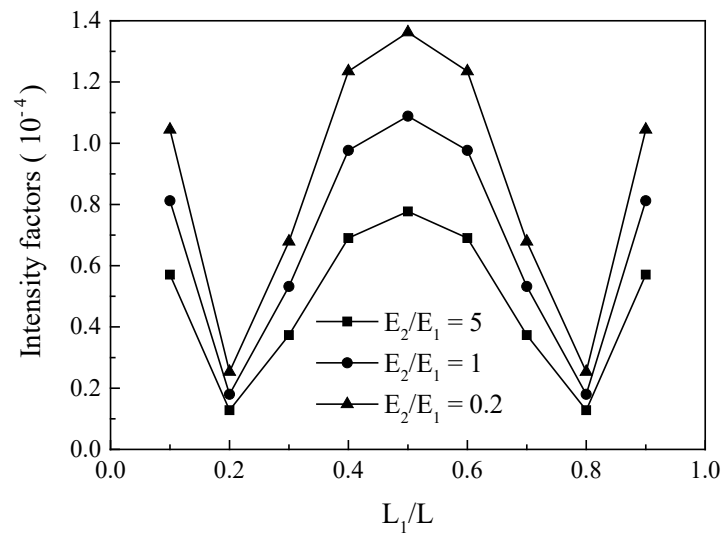

(a)

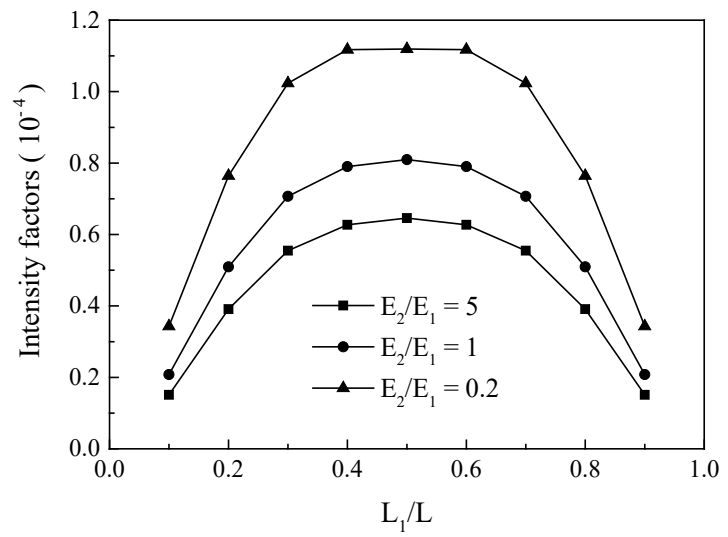

(b)

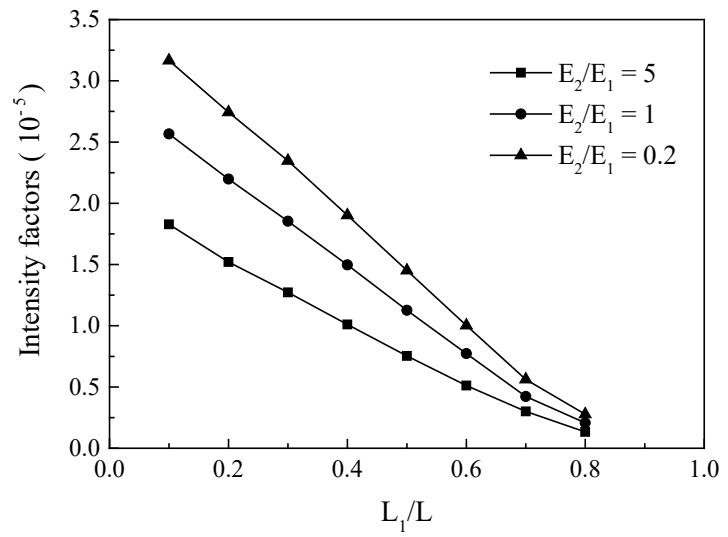

(c)

Fig.7. Effect of the Young's modulus ratio on the intensity factor of edge cracked FGM beams with $a / h=0.2$ versus crack position: (a) C-C beam; (b) H-H beam; (c) C-F beam. 


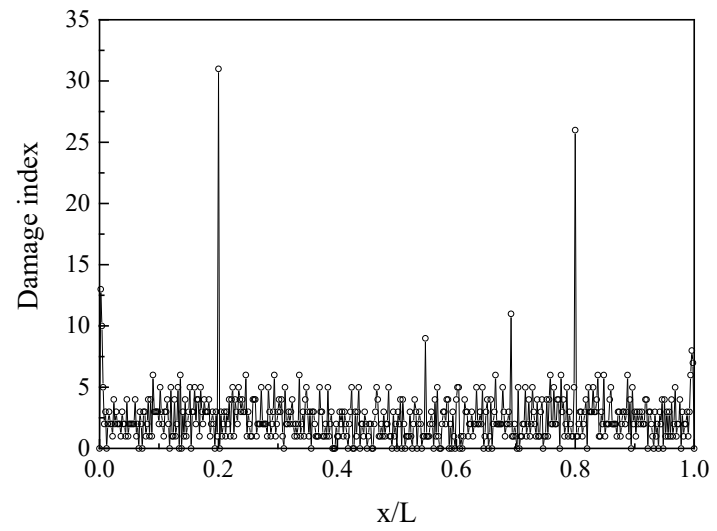

(a)

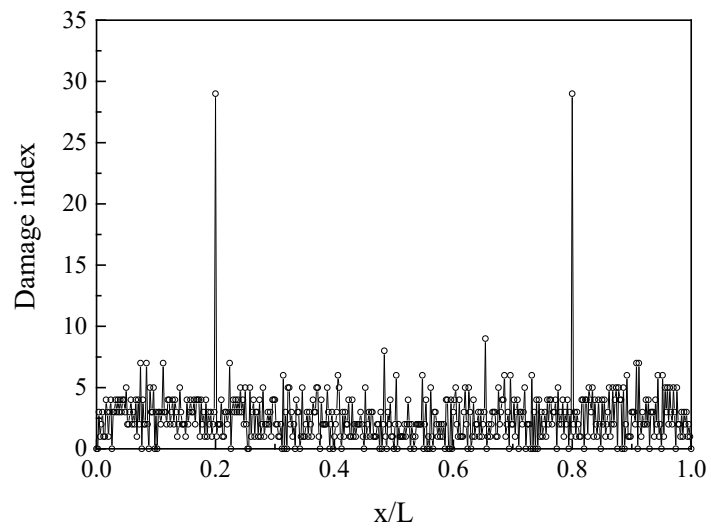

(b)

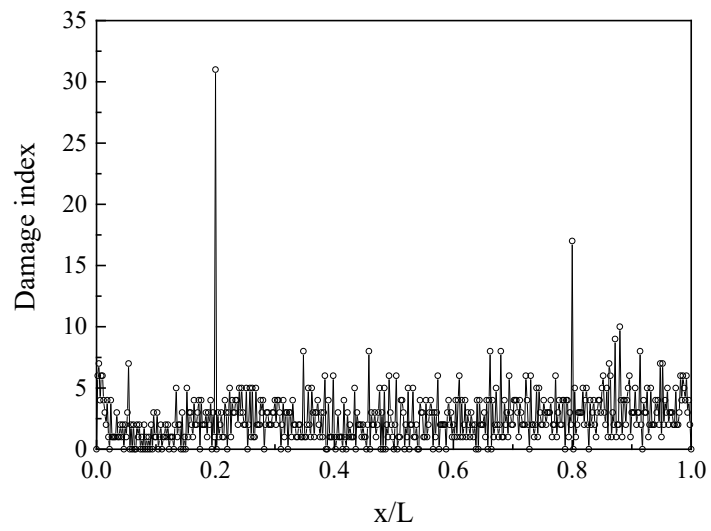

(c)

Fig.8. Damage index of the FGM beam with two edge cracks $\left(E_{2} / E_{1}=5, L_{1} / L=0.2, L_{2} / L=0.8\right.$, $a_{1} / h=0.3, a_{2} / h=0.2$ ): (a) C-C beam; (b) H-H beam; (c) C-F beam. 


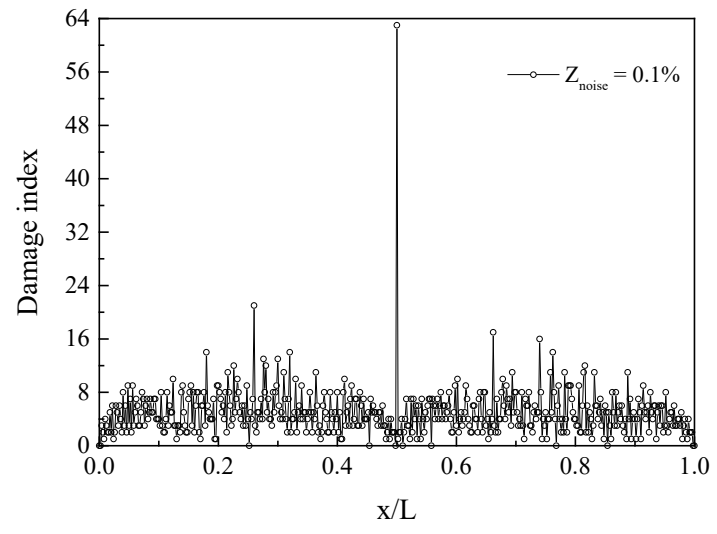

(a)

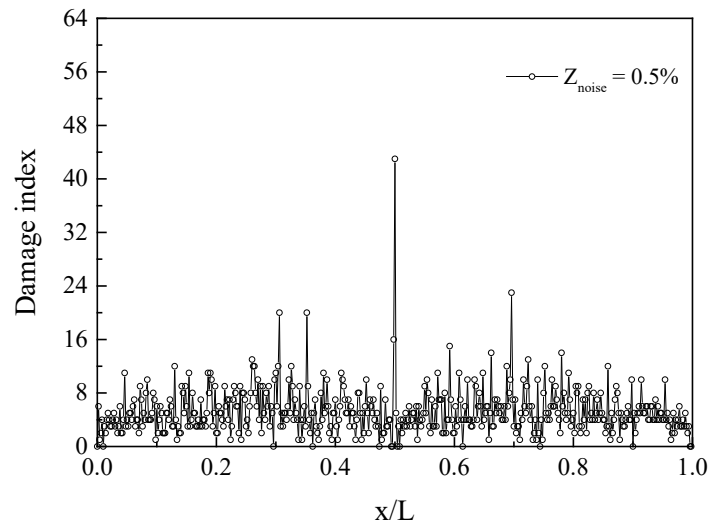

(b)

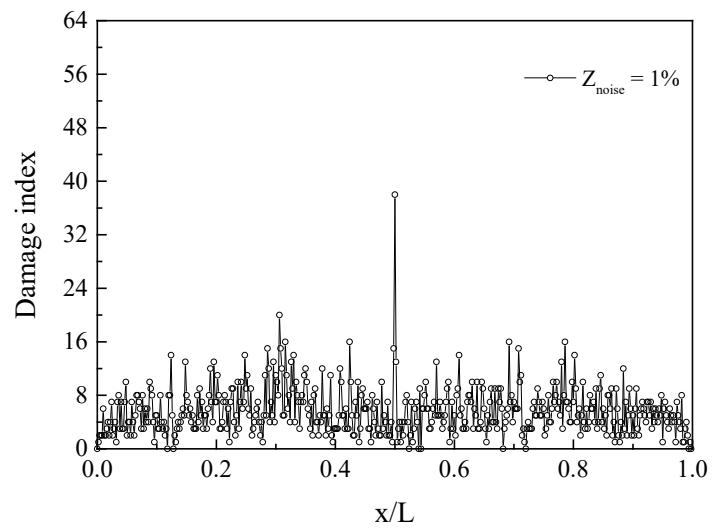

(c)

Fig. 9 Damage index of the H-H cracked FGM beam with single crack $\left(E_{2} / E_{1}=5, L_{1} / L=0.5\right.$, $a_{1} / h=0.3$ ) in noisy conditions: (a) $Z_{\text {noise }}=0.1 \%$; (b) $Z_{\text {noise }}=0.5 \%$; (c) $Z_{\text {noise }}=1 \%$. 